\title{
Extension of competition graphs under complex fuzzy environment
}

\author{
Muhammad Akram $^{1} \cdot$ Aqsa Sattar $^{1} \cdot$ Faruk Karaaslan $^{2}$ (I) $\cdot$ Sovan Samanta $^{3}$
}

Received: 3 May 2020 / Accepted: 7 October 2020 / Published online: 4 November 2020

(C) The Author(s) 2020

\begin{abstract}
A complex fuzzy set (CFS) is a remarkable generalization of the fuzzy set in which membership function is restricted to take the values from the unit circle in the complex plane. A CFS is an efficient model to deal with uncertainties of human judgement in more comprehensive and logical way due to the presence of phase term. In this research article, we introduce the concept of competition graphs under complex fuzzy environment. Further, we present complex fuzzy $k$-competition graphs and $p$-competition complex fuzzy graphs. Moreover, we consider $m$-step complex fuzzy competition graphs, complex fuzzy neighborhood graphs (CFNGs), complex fuzzy economic competition graphs (CFECGs) and $m$-step complex fuzzy economic competition graphs with interesting properties. In addition, we describe an application in ecosystem of our proposed model. We also provide comparison of proposed competition graphs with existing graphs.
\end{abstract}

Keywords Complex fuzzy set $\cdot k$-Competition $\cdot p$-Competition $\cdot m$-Step competition $\cdot$ Economic competition.

\section{Introduction}

In 1968, Cohen [6] introduced the conception of competition graphs (CGs) to determine the problems of ecosystem. CGs have many utilizations in distinct areas of life. The analogy of Cohen was based on the fact that there is a competition between two species if both species have a common prey. Many variations of CGs are present in the literature, namely competition common enemy graph of digraph [22], $p$-competition graphs of digraph $[12,13]$, competition hypergraphs [23] and tolerance competition graphs [4]. Another worthwhile generalization of competition graphs was given by Cho et al. [5] in 2000. All the introduced competi-

Faruk Karaaslan

karaaslan.faruk@gmail.com

Muhammad Akram

m.akram@pucit.edu.pk

Aqsa Sattar

sattaraqsa47@gmail.com

Sovan Samanta

ssamantavu@gmail.com

1 Department of Mathematics, University of the Punjab, New Campus, Lahore 54590, Pakistan

2 Department of Mathematics, Faculty of Sciences, Çankiri Karatekin University, 18100, Çankiri, Turkey

3 Department of Mathematics, Tamralipta Mahavidyalaya, Tamluk, West Bengal 721636, India tion graphs are crisp graphs which cannot describe all the real-world competitions. In ecosystem, the species may be vegetarian, non-vegetarian, strong, weak, and similarly the prey may be harmful, digestive, energetic, etc.

In 1965, Zadeh [25] developed the conception of fuzzy set to represent the uncertainty of human judgment by initiating the membership function which is restricted to take the values form the unit interval $[0,1]$. For example, today is sunny, it might be $100 \%$ true if there are no clouds, $80 \%$ true if there are few clouds, $50 \%$ true if it is hazy and $0 \%$ true if it rains all day. A graph is an easy way to interpret the information which involve the relationship between objects. Fuzzy graphs are designed to represent the structures of relationships between objects such that the existence of a concrete object (vertex) and the relationship between two objects (edge) are matters of degree. In 1975, Rosenfeld [17] initiated the notion of fuzzy graph (FG). Certain notions of FGs have been discussed in $[1,8,15,27]$. After the introduction of FG, many researchers turned their attention to find the competition among entities under fuzzy environment. Fuzzy $k$-competition and $p$-competition graphs were developed by Samanta and Pal [20]. Further, Samanta et al. [19] initiated $m$-step fuzzy competition graphs. Moreover, Habib et al. [9] initiated the concept of q-rung orthopair FCGs with their utilization in soil ecosystem. Sarwar et al. [21] introduced a new decision-making approach with fuzzy competition hypergraphs. Akram and Luqman [2] presented 
a remarkable contribution on fuzzy hypergraphs. Most of the real-world competitions can be represented through these FCGs but there exist some competitions in which the entities possess the periodic or two-dimensional information which cannot be represented through these FCGs. For example, in a food web, the species may be strong and vegetarian and the prey may be harmful and energetic at the same time interval. Similarly, the species may be strong or weak and the prey may be harmful or energetic under some specific time interval. This information about the species and preys is two dimensional which cannot be modeled through FCGs.

Fuzzy set (FS) theory is useful model to deal with uncertainties arising in various fields of life. But in some situations, this theory cannot model the incomplete and imprecise information of two-dimensional or periodic nature encountered in the real world. To cope with this problem, Ramot et al. [16] proposed complex fuzzy set (CFS), as a worthwhile generalization of FS in which the membership grade is restricted to take the values form the unit circle in the complex plane, and is of the form $r e^{i \theta}$, where $r$ is the amplitude term and $\theta$ is the phase term. The phase term $\theta$ of CFS has a great importance because it can handle the recurring problems or periodic problems phenomena with more perfection. The presence of phase term in CFS ensures that there may exist some situations where the second dimension is required. This term differentiates CFS from all other available sets of the literature. Possible application which demonstrates the new concept involve a CF representation of solar activity, signal processing application and in time series forecasting applications. Later on, Thirunavukarasu [24] introduced the idea of complex fuzzy graph (CFG). Recently, Luqman et al. [14] investigated the novel concepts of CF hypergraphs. Akram et al. [3] considered imperfect competition models in economic market structure with $q$-rung picture fuzzy information. Further, Garg and Rani $[10,11]$ investigated many decision-making techniques. The motivation to this article can be described as follows.

1. The CFS, a worthwhile generalization of FS, is a proficient model to handle all the periodic or two-dimensional information due to the presence of an additional term known as phase term. This set has an edge over the other existing models of the literature due to its additional features of handling the periodic and two-dimensional information in a single set. On the other hand, if we remove the phase term of CFS then the CFS is a conventional FS with real-valued membership.

2. The competition graphs developed under $\mathrm{CF}$ environment are useful enough to tackle all the competitions of real world which possess the information of twodimensional nature.
In this research article, we present the innovative concept of CFCGs. We then investigate the two generalizations of CFCGs, namely CF $k$-competition and $p$-competition CFGs. Further, we investigate CFNGs, $m$-step CFCGs, CFECGs and $m$-step CFECGs. Moreover, we discuss an application of CFCGs in ecosystem to emphasize the superiority of these graphs in real life. The main contribution to this article is summarized as follows.

1. Competition graphs with its remarkable generalization are developed in CF environment to overcome the deficiencies of other existing competition graphs of the literature.

2. An algorithm is initiated to find the competition among the real-world entities with an application in ecology.

3. A comparative analysis is provided to check the superiority and authenticity of our proposed competition graphs.

We arrange this paper as follows: the next section includes some elemental definitions which are necessary for the evolution of CFCGs, followed by which we introduce the concept of CFCGs with its two remarkable extensions, namely CF $\mathrm{k}$-competition graphs and p-competition CFGs. In the subsequent section, we develop the concept of CFNGs with some related theorems and then we discuss $m$-step CFCGs. After this, we initiate a new concept called CFECGs. The penultimate section includes an application of CFCG to reveal its importance in real life. Before the concluding section, we provide comparison of the proposed competition graphs with existing graphs. Finally, we give the conclusion.

\section{Preliminaries}

Definition 1 [20] The fuzzy competition graph (FCG) $\mathfrak{C}(\vec{\Omega})$ of fuzzy digraph (FDG) $\vec{\Omega}=(W, \widehat{\mathbb{P}}, \widehat{\widehat{Q}})$ is an undirected graph $\Omega=(W, \widehat{\mathbb{P}}, \widehat{\mathbb{Q}})$ with same node set as in $\vec{\Omega}$ and have an edge connecting two distinct vertices $g$ and $f$ in $\mathfrak{C}(\vec{\Omega})$ if and only if $\aleph^{+}(g) \cap \aleph^{+}(f) \neq \varnothing$ in $\vec{\Omega}$, and for the edge $(g, f)$ the membership value (MV) in $C(\vec{\Omega})$ is given as

$\xi_{\widehat{\mathbb{Q}}}(g, f)=\left(\xi_{\widehat{\mathbb{P}}}(g) \wedge \xi_{\widehat{\mathbb{P}}}(f)\right) \times \hbar\left(\aleph^{+}(g) \cap \aleph^{+}(f)\right)$.

Definition 2 [16] Let $Z$ be a universal set. Then complex fuzzy set (CFS) $B$ on $Z$ can be given as

$B=\left\{\left(q, \xi_{B}(q) e^{i \varphi_{B}(q)}\right) \mid q \in Z\right\}$,

where $\xi_{B}(q) e^{i \varphi_{B}(q)}$ is the MV of the element $q$ such that $\xi_{B}(q) \in[0,1]$ and $\varphi_{B}(q) \in[0,2 \pi]$, and $i=\sqrt{-1}$. For every $q \in Z, \xi_{B}(q)$ and $\varphi_{B}(q)$ are the amplitude term and phase term, respectively. 
Definition 3 [7] A complex fuzzy relation (CFR) $\mathbb{R}$ on a universal set $W \times Z$, can be given as

$\mathbb{R}(g, f)=\left\{\left((g, f), \xi_{\mathbb{R}}(g, f) e^{i \varphi_{\mathbb{R}}(g, f)}\right) \mid(g, f) \in W \times Z\right\}$,

where $\xi_{\mathbb{R}}(g, f) e^{i \varphi_{\mathbb{R}}(g, f)}$ is the MV of the element $(g, f)$ in $W \times Z$ such that $\xi_{\mathbb{R}}(g, f) \in[0,1]$ and $\varphi_{\mathbb{R}}(g, f) \in[0,2 \pi]$, and $i=\sqrt{-1}$.

For each $(g, f) \in W \times Z, \xi_{R}(g, f)$ and $\varphi_{R}(g, f)$ are the amplitude term and the phase term, respectively.

Definition 4 [26] Let $B_{1}$ and $B_{2}$ be two CFSs on a universal set $Z$, where

$B_{1}=\left\{q, \xi_{B_{1}}(q) e^{i \varphi_{B_{1}}(q)} \mid q \in Z\right\}$,

$B_{2}=\left\{q, \xi_{B_{2}}(q) e^{i \varphi_{B_{2}}(q)} \mid q \in Z\right\}$.

Then the CF intersection of $B_{1}$ and $B_{2}$, symbolized by $B_{1} \cap$ $B_{2}$, is given by the function

$B_{1} \cap B_{2}=\left\{\xi_{B_{1} \cap B_{2}}(q) e^{i \varphi_{B_{1} \cap B_{2}}(q)} \mid q \in Z\right\}$,

where

$\xi_{B_{1} \cap B_{2}}(q) e^{i \varphi_{B_{1} \cap B_{2}}(q)}=\left(\xi_{B_{1}}(q) \wedge \xi_{B_{2}}(q)\right) e^{i \varphi_{B_{1}}(q) \wedge \varphi_{B_{2}}(q)}$.

Definition 5 [26] Let $B_{1}$ and $B_{2}$ be two CFSs defined on a universe $Z$, where

$B_{1}=\left\{q, \xi_{B_{1}}(q) e^{i \varphi_{B_{1}}(q)} \mid q \in Z\right\}$,

$B_{2}=\left\{q, \xi_{B_{2}}(q) e^{i \varphi_{B_{2}}(q)} \mid q \in Z\right\}$.

Then the CF product of $B_{1}$ and $B_{2}$, symbolized by $B_{1} \circ B_{2}$, is given by the function

$B_{1} \circ B_{2}=\left\{\xi_{B_{1} \circ B_{2}}(q) e^{i \varphi_{B_{1} \circ B_{2}}(q)} \mid q \in Z\right\}$,

where where

$\left.\xi_{B_{1} \circ B_{2}}(q) e^{i \varphi_{B_{1} \circ B_{2}}(q)}=\left(\xi_{B_{1}}(q) \cdot \xi_{B_{2}}(q)\right) e^{i 2 \pi\left(\frac{\varphi_{B_{1}}(q)}{2 \pi} \cdot \frac{\varphi_{B_{2}}(q)}{2 \pi}\right.}\right)$

Definition 6 [24] A fuzzy graph $(\mathrm{FG}) \Omega=(W, \widehat{\mathbb{P}}, \widehat{\mathbb{Q}})$, on a non-empty set $W$ is said to be a complex fuzzy graph (CFG), where $\widehat{\mathbb{P}}$ and $\widehat{\mathbb{Q}}$ are CFS and CFR on $W$ such that

$\xi_{\widehat{\mathbb{Q}}}(g, f) \leq\left(\xi_{\widehat{\mathbb{P}}}(g) \wedge \xi_{\widehat{\mathbb{P}}}(f)\right), \quad$ (for amplitude term)

$\varphi_{\widehat{\mathbb{Q}}}(g, f) \leq\left(\varphi_{\widehat{\mathbb{P}}}(g) \wedge \xi_{\widehat{\mathbb{P}}}(f)\right), \quad($ for phase term $)$

for all $g, f \in W$.
Table 1 List of acronyms

\begin{tabular}{ll}
\hline Acronyms & Description \\
\hline FS & Fuzzy set \\
CFS & Complex fuzzy set \\
CFON & Complex fuzzy out-neighborhood \\
CFIN & Complex fuzzy in-neighborhood \\
CFCG & Complex fuzzy competition graph \\
$C F O^{p} N$ & Complex fuzzy open neighborhood \\
$C F C^{l} N$ & Complex fuzzy closed neighborhood \\
$C F O^{p} N G$ & Complex fuzzy open neighborhood graph \\
$C F C^{l} N G$ & Complex fuzzy closed neighborhood graph \\
$\mathrm{CFECG}$ & Complex fuzzy economic competition graph
\end{tabular}

Definition 7 [24] A directed CFG (complex fuzzy digraph) (CFDG) on a non-empty set $W$ is a triplet $\vec{\Omega}=(W, \widehat{\mathbb{P}}, \overrightarrow{\mathbb{Q}})$, where $\widehat{\mathbb{P}}$ is $\mathrm{CFS}$ on $W$ and $\overrightarrow{\mathbb{Q}}$ is CFR on $W$ such that

$\xi_{\widehat{\mathbb{Q}}} \overrightarrow{(g, f)} \leq\left(\xi_{\widehat{\mathbb{P}}}(g) \wedge \xi_{\widehat{\mathbb{P}}}(f)\right)$, (for amplitude term)

$\varphi_{\widehat{\mathbb{Q}}} \overrightarrow{(g, f)} \leq\left(\varphi_{\widehat{\mathbb{P}}}(g) \wedge \xi_{\widehat{\mathbb{P}}}(f)\right), \quad$ (for phase term)

for all $g, f \in W$.

The list of acronyms in research paper is given in Table 1.

\section{Complex fuzzy set applied to competition graphs}

This section presents some basic definitions which are necessary to define for the evolution CFCGs.

Definition 8 For a node $g$ of a CFDG $\vec{\Omega}=(W, \widehat{\mathbb{P}}, \overrightarrow{\mathbb{Q}})$, the $\mathrm{CF}$ out-neighborhood (CFON) is a CFS, given by

$\aleph^{p}(g)=\left(S_{g}^{p}, m_{g}^{p} e^{i \eta_{g}^{p}}\right)$,

where $S_{g}^{p}=\left\{f \mid \xi_{\widehat{\mathbb{Q}}} \overrightarrow{(g, f)}>0\right\}$ such that $m_{g}^{p}: S_{g}^{p} \rightarrow[0,1]$ given by $m_{g}^{p}(f)=\xi_{\widehat{Q}} \overrightarrow{(g, f)}$ and $\eta_{g}^{p}: S_{g}^{p} \rightarrow[0,2 \pi]$ given by $\eta_{g}^{p}(f)=\varphi_{\widehat{\mathbb{Q}}} \overrightarrow{(g, f)}$.

Definition 9 For a node $g$ of a CFDG $\vec{\Omega}=(W, \widehat{\mathbb{P}}, \overrightarrow{\mathbb{Q}})$, the $\mathrm{CF}$ in-neighborhood (CFIN) is a CFS, given by

$\aleph^{n}(g)=\left(S_{g}^{n}, m_{g}^{n} e^{i \eta_{g}^{n}}\right)$, 


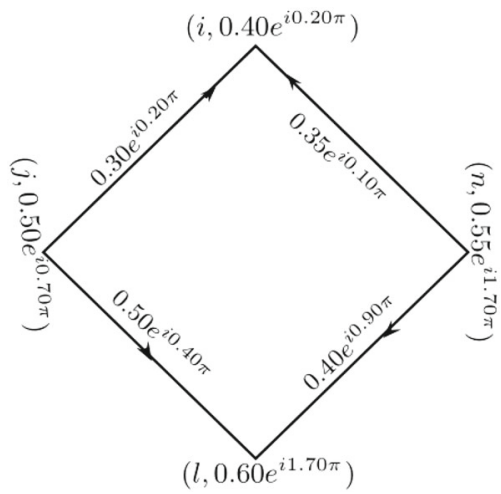

Fig. 1 Complex fuzzy digraph

where $S_{g}^{n}=\left\{f \mid \xi_{\widehat{Q}} \overrightarrow{(f, g)}>0\right\}$ such that $m_{g}^{n}: S_{g}^{n} \rightarrow[0,1]$ given by $m_{g}^{n}(f)=\xi_{\widehat{Q}}\left(\overrightarrow{f, g)}\right.$ and $\eta_{g}^{n}: S_{g}^{n} \rightarrow[0,2 \pi]$ given by $\eta_{g}^{n}(f)=\varphi_{\widehat{Q}} \overrightarrow{(f, g)}$.

Example 1 Let $\vec{\Omega}=(W, \widehat{\mathbb{P}}, \overrightarrow{\mathbb{Q}})$ be a CFDG, where

$$
\begin{aligned}
& \widehat{\mathbb{P}}=\left\langle\frac{i}{0.40 e^{i 0.20 \pi}}, \frac{j}{0.50 e^{i 0.70 \pi}}, \frac{l}{0.60 e^{i 1.70 \pi}},\right. \\
& \left.\frac{n}{0.55 e^{i 1.70 \pi}}\right\rangle, \\
& \overrightarrow{\widehat{\mathbb{Q}}}=\left\langle\frac{\overrightarrow{(j, i)}}{0.30 e^{i 0.20 \pi}}, \frac{\overrightarrow{(n, i)}}{0.350 e^{i 0.10 \pi}}, \frac{\overrightarrow{(j, l)}}{0.50 e^{i 0.40 \pi}},\right. \\
& \left.\frac{\overrightarrow{(n, l)}}{0.40 e^{i 0.90 \pi}}\right\rangle .
\end{aligned}
$$

The CFDG is shown in Fig. 1.

CFON and CFIN of the nodes are given in Table 2.

Definition 10 Let $B=\left\{\left(q, \xi_{B}(q) e^{i \varphi_{B}(q)}\right) \mid q \in Z\right\}$ be a CFS. The height of CFS $B$ symbolized by $\hbar(B)=\left(\hbar_{\xi}(B) e^{i \hbar_{\varphi}}\right)$ is given as

$$
\begin{aligned}
\hbar(B) & =\left\{\left(\max \left(\xi_{B}(q)\right) e^{i \max \left(\varphi_{B}(q)\right)}\right) \mid q \in Z\right\} \\
& =\left(\hbar_{\xi}(B) e^{i \hbar_{\varphi}(B)}\right) .
\end{aligned}
$$

Definition 11 The CFCG $\mathfrak{C}(\vec{\Omega})$ of a CFDG $\vec{\Omega}=$ $(W, \widehat{\mathbb{P}}, \overrightarrow{\mathbb{Q}})$ is an undirected $\mathrm{CFG} \Omega=(W, \widehat{\mathbb{P}}, \widehat{\mathbb{Q}})$ with same CF node set as in $\vec{\Omega}$ and possesses a CF edge in $\mathfrak{C}(\vec{\Omega})$ joining two distinct nodes $g$ and $f$ if and only if the CFS $\aleph^{p}(g) \cap \aleph^{p}(f) \neq \varnothing$ in $\vec{\Omega}$ and the MV of the edge $(g, f)$ in $\mathfrak{C}(\vec{\Omega})$ is

$$
\begin{aligned}
\xi_{\widehat{\mathbb{Q}}}(g, f)= & \left(\xi_{\widehat{\mathbb{P}}}(g) \wedge \xi_{\widehat{\mathbb{P}}}(f)\right) \times \hbar_{\xi}\left(\aleph^{p}(g) \cap \aleph^{p}(f)\right), \\
\varphi_{\widehat{\mathbb{Q}}}(g, f)= & 2 \pi\left[\left(\frac{\varphi_{\widehat{\mathbb{P}}}(g)}{2 \pi} \wedge \frac{\varphi_{\widehat{\mathbb{P}}}(f)}{2 \pi}\right)\right. \\
& \left.\times \frac{\hbar_{\varphi}\left(\aleph^{p}(g) \cap \aleph^{p}(f)\right)}{2 \pi}\right] .
\end{aligned}
$$

Example 2 Let $\vec{\Omega}=(W, \widehat{\mathbb{P}}, \overrightarrow{\mathbb{Q}})$ be a CFDG, as presented in Fig. 2a, given by

$$
\begin{aligned}
& \widehat{\mathbb{P}}=\left\langle\frac{l}{0.3 e^{i 0.7 \pi}}, \frac{i}{0.6 e^{i 1.3 \pi}}, \frac{m}{0.4 e^{0.4 e^{i 1.2 \pi}}}, \frac{k}{0.5 e^{i 0.9 \pi}},\right. \\
& \left.\frac{n}{0.4 e^{i 1.7 \pi}}\right\rangle, \\
& \overrightarrow{\widehat{\mathbb{Q}}}=\left\langle\frac{\overrightarrow{(i, l)}}{0.2 e^{i 0.3 \pi}}, \frac{\overrightarrow{(m, i)}}{0.35 e^{i 1.1 \pi}}, \frac{\overrightarrow{(k, m)}}{0.2 e^{i 0.7 \pi}}, \frac{\overrightarrow{(n, k)}}{0.2 e^{i 0.7 \pi}},\right. \\
& \left.\frac{\overrightarrow{(n, l)}}{0.25 e^{i 0.2 \pi}}, \frac{\overrightarrow{(k, l)}}{0.3 e^{i 0.2 \pi}}\right\rangle .
\end{aligned}
$$

The CFON of the nodes are shown in Table 3.

The CFSs $\aleph^{p}(g) \cap \aleph^{p}(f)$ and $\hbar\left(\aleph^{p}(g) \cap \aleph^{p}(f)\right)$ are given in Table 4.

The corresponding CFCG is shown in Fig. $2 b$.

Definition 12 Let $B=\left\{\left(q, \xi_{B}(q)\right) e^{i \varphi_{B}(q)} \mid q \in Z\right\}$ be a CFS. Then the cardinality of CFS $B$ is a positive complex number symbolized by $|B|_{\xi} e^{i|B|_{\varphi}}$, is defined as

$$
\begin{aligned}
|B| & =\sum_{q_{i} \in Z} \xi_{B}\left(q_{i}\right) e^{i \sum_{q_{i} \in Z} \varphi_{B}\left(q_{i}\right)} \\
& =|B| \xi e^{i|B|_{\varphi}},
\end{aligned}
$$

for all $q \in Z$.

Definition 13 Suppose $k=r e^{i \theta}$ be a complex number and $\left|\aleph^{p}(g) \cap \aleph^{p}(f)\right|=r^{\prime} e^{i \theta^{\prime}}($ say $)$. The CF $k$-competition graph $\mathfrak{C}_{k}(\vec{\Omega})$ of a CFDG $\vec{\Omega}=(W, \widehat{\mathbb{P}}, \overrightarrow{\mathbb{Q}})$ is an undirected CFG $\Omega=(W, \widehat{\mathbb{P}}, \widehat{\mathbb{Q}})$ with same $\mathrm{CF}$ node set as in $\vec{\Omega}$ and have a CF edge in $\mathfrak{C}_{k}(\vec{\Omega})$ connecting two distinct vertices $g$ and $f$
Table 2 CFON and CFIN of the nodes

\begin{tabular}{lll}
\hline $\mathrm{g}$ & $\aleph^{p}(g)$ & $\aleph^{n}(g)$ \\
\hline $\mathrm{i}$ & $\varnothing$ & $\left\{\left(j, 0.3 e^{i 0.20 \pi}\right),\left(n, 0.35 e^{i 0.10 \pi}\right)\right\}$ \\
$\mathrm{j}$ & $\left\{\left(l, 0.50 e^{i 0.40 \pi}\right),\left(i, 0.30 e^{i 0.20 \pi}\right)\right\}$ & $\varnothing$ \\
$\mathrm{l}$ & $\varnothing$ & $\left\{\left(n, 0.40 e^{i 0.90 \pi}\right),\left(j, 0.5 e^{i 0.40 \pi}\right)\right\}$ \\
$\mathrm{n}$ & $\left\{\left(l, 0.40 e^{i 0.90 \pi}\right),\left(i, 0.35 e^{i 0.10 \pi}\right)\right\}$ & $\varnothing$ \\
\hline
\end{tabular}


Fig. 2 Complex fuzzy digraph and complex fuzzy competition graph

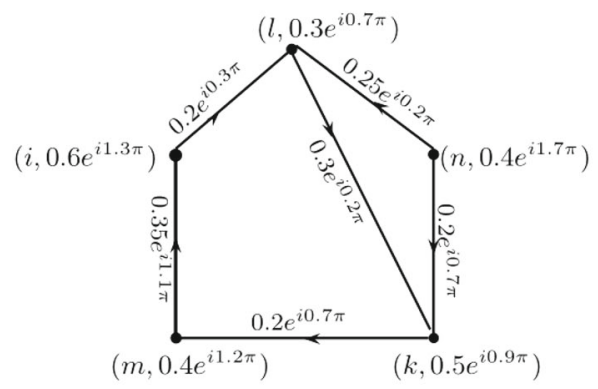

$\vec{\Omega}$

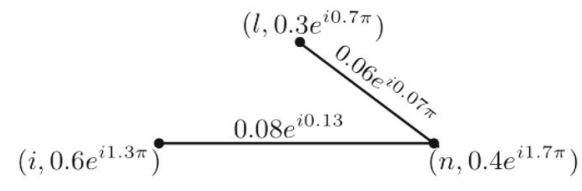

$\left(m, 0.4 e^{\mathfrak{i}_{1.2 \pi}}\right)$

$\left(k, 0.5 e^{i 0.9 \pi}\right)$

(a) CFDG

(b) CFCG

Table 3 CFON of the nodes

\begin{tabular}{ll}
\hline $\mathrm{g}$ & $\aleph^{p}(g)$ \\
\hline 1 & $\left\{\left(k, 0.3 e^{i 0.2 \pi}\right)\right\}$ \\
$\mathrm{i}$ & $\left\{\left(l, 0.2 e^{i 0.3 \pi}\right)\right\}$ \\
$\mathrm{m}$ & $\left\{\left(i, 0.35 e^{i 1.1 \pi}\right)\right\}$ \\
$\mathrm{k}$ & $\left\{\left(m, 0.2 e^{i 0.7 \pi}\right)\right\}$ \\
$\mathrm{n}$ & $\left\{\left(l, 0.25 e^{i 0.2 \pi}\right),\left(k, 0.2 e^{i 0.7 \pi}\right)\right\}$ \\
\hline
\end{tabular}

if and only if $r^{\prime}>r$ and $\theta^{\prime}>\theta$. The MV of the edge $(g, f)$ in $\mathfrak{C}_{k}(\vec{\Omega})$ is calculated as

$\xi_{\widehat{\mathbb{Q}}}(g, f)=\left(\frac{r^{\prime}-r}{r^{\prime}}\right)\left(\xi_{\widehat{\mathbb{P}}}(g) \wedge \xi_{\widehat{\mathbb{P}}}(f)\right) \times \hbar_{\xi}\left(\aleph^{p}(g) \cap \aleph^{p}(f)\right)$,

$\varphi_{\widehat{\mathbb{Q}}}(g, f)=2 \pi\left[\frac{\theta^{\prime}-\theta}{\theta^{\prime}}\left(\frac{\varphi_{\widehat{\mathbb{P}}}(g)}{2 \pi} \wedge \frac{\varphi_{\widehat{\mathbb{P}}}(f)}{2 \pi}\right) \times \frac{\hbar_{\varphi}\left(\aleph^{p}(g) \cap \aleph^{p}(f)\right)}{2 \pi}\right]$.

Example 3 Let $\vec{\Omega}=(W, \widehat{\mathbb{P}}, \overrightarrow{\mathbb{Q}})$ be a CFG as presented in Fig. 3, given by

$$
\begin{aligned}
& \widehat{\mathbb{P}} \quad=\quad\left\langle\frac{l}{0.6 e^{i i^{i 1.2 \pi}}}, \frac{j}{0.4 e^{i 1.2 \pi}}, \frac{x}{0.6 e^{i 0.3 \pi}},\right. \\
& \left.\frac{z}{0.7 e^{i 1.3 \pi}}, \frac{m}{0.8 e^{i 0.9 \pi}}, \frac{n}{0.65 e^{i 1 \pi}}\right\rangle, \\
& \overrightarrow{\mathbb{Q}}=\left\langle\frac{\overrightarrow{(l, x)}}{0.6 e^{i 0.3 \pi}}, \frac{\overrightarrow{(l, z)}}{0.5 e^{i 0.2 \pi}}, \frac{\overrightarrow{(l, m)}}{0.45 e^{i 0.3 \pi}}, \frac{\overrightarrow{(l, n)}}{0.35 e^{i 0.2 \pi}},\right. \\
& \left.\frac{\overrightarrow{(j, x)}}{0.3 e^{i 0.2 \pi}}, \frac{\overrightarrow{(j, z)}}{0.35 e^{i 0.9 \pi}}, \frac{\overrightarrow{(j, m)}}{0.36 e^{i 0.2 \pi}}, \frac{\overrightarrow{(j, n)}}{0.4 e^{i 1 \pi}}\right\rangle \text {. }
\end{aligned}
$$

The CFON of the nodes are given in Table 5 .

Next, we have

$$
\begin{aligned}
& \aleph^{p}(j) \cap \aleph^{p}(l) \\
& \quad=\left\{\left(n, 0.35 e^{i 0.2 \pi}\right),\left(m, 0.36 e^{i 0.2 \pi}\right),\left(z, 0.35 e^{i 0.2 \pi}\right),\left(x, 0.3 e^{i 0.2 \pi}\right)\right\} .
\end{aligned}
$$

Table 4 The CFS $\aleph^{p}(g) \cap \aleph^{p}(f)$ and $\hbar\left(\aleph^{p}(g) \cap \aleph^{p}(f)\right)$

\begin{tabular}{llll}
\hline $\mathrm{g}$ & $\mathrm{f}$ & $\aleph^{p}(g) \cap \aleph^{p}(f)$ & $\hbar\left(\aleph^{p}(g) \cap \aleph^{p}(f)\right)$ \\
\hline 1 & $\mathrm{i}$ & $\varnothing$ & $\varnothing$ \\
1 & $\mathrm{~m}$ & $\varnothing$ & $\varnothing$ \\
1 & $\mathrm{k}$ & $\varnothing$ & $\varnothing$ \\
1 & $\mathrm{n}$ & $\left\{\left(k, 0.2 e^{i 0.2 \pi}\right)\right\}$ & $\left\{\left(0.2 e^{i 0.2 \pi}\right)\right\}$ \\
$\mathrm{i}$ & $\mathrm{m}$ & $\varnothing$ & $\varnothing$ \\
$\mathrm{i}$ & $\mathrm{k}$ & $\varnothing$ & $\varnothing$ \\
$\mathrm{i}$ & $\mathrm{n}$ & $\left\{\left(l, 0.2 e^{i 0.2 \pi}\right)\right\}$ & $\left\{\left(0.2 e^{i 0.2 \pi}\right)\right\}$ \\
$\mathrm{m}$ & $\mathrm{k}$ & $\varnothing$ & $\varnothing$ \\
$\mathrm{m}$ & $\mathrm{n}$ & $\varnothing$ & $\varnothing$ \\
$\mathrm{k}$ & $\mathrm{n}$ & $\varnothing$ & $\varnothing$ \\
\hline
\end{tabular}

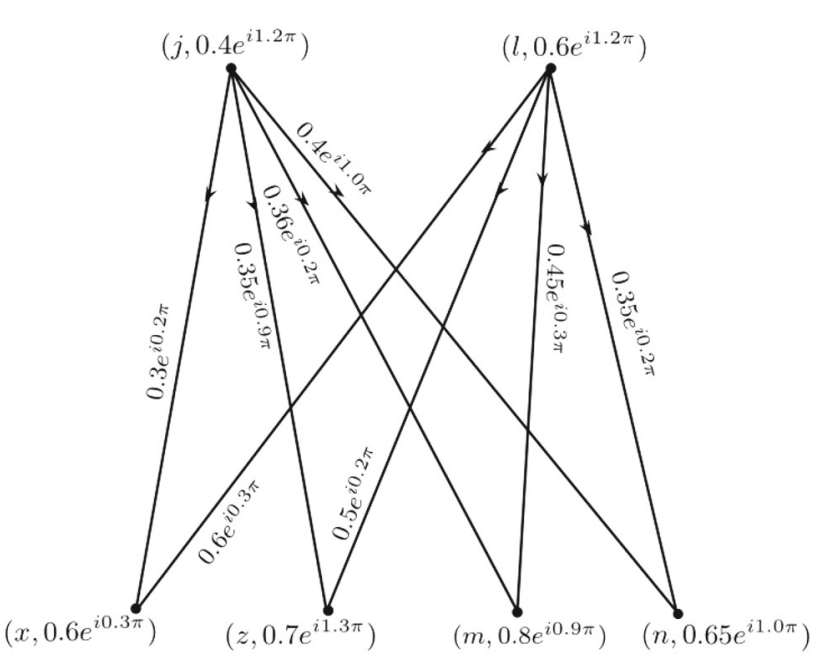

Fig. 3 Complex fuzzy digraph

The CFSs $\hbar\left(\aleph^{p}(j) \cap \aleph^{p}(l)\right)$ and $\left|\left(\aleph^{p}(j) \cap \aleph^{p}(l)\right)\right|$ are

$$
\begin{aligned}
& \hbar\left(\aleph^{p}(j) \cap \aleph^{p}(l)\right) \\
& =\left\{\left(0.36 e^{i 0.2 \pi}\right)\right\}, \quad\left|\left(\aleph^{p}(j) \cap \aleph^{p}(l)\right)\right|=\left(1.36 e^{i 0.8 \pi}\right) .
\end{aligned}
$$


Table 5 The CFON of the nodes

\begin{tabular}{ll}
\hline $\mathrm{g}$ & $\aleph^{p}(g)$ \\
\hline $\mathrm{j}$ & $\left\{\left(n, 0.4 e^{i 1.0 \pi}\right),\left(m, 0.36 e^{i 0.2 \pi}\right),\left(x, 0.3 e^{i 0.2 \pi}\right),\left(z, 0.35 e^{i 0.9 \pi}\right)\right\}$ \\
$\mathrm{l}$ & $\left\{\left(n, 0.35 e^{i 0.2 \pi}\right),\left(m, 0.45 e^{i 0.3 \pi}\right),\left(x, 0.6 e^{i 0.3 \pi}\right),\left(z, 0.5 e^{i 0.2 \pi}\right)\right\}$ \\
$\mathrm{x}$ & $\varnothing$ \\
$\mathrm{z}$ & $\varnothing$ \\
$\mathrm{m}$ & $\varnothing$ \\
$\mathrm{n}$ & $\varnothing$ \\
\hline
\end{tabular}

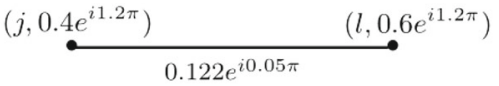

$\left(x, 0.6 e^{i 0.3 \pi}\right) \quad\left(z, 0.7 e^{i 1.3 \pi}\right) \quad\left(m, \dot{0.8 e^{i 0.9 \pi}}\right) \quad\left(n, \dot{0} 0.65 e^{i 1.0 \pi}\right)$

Fig. $4 \mathrm{CF}-0.2 e^{i 0.2 \pi}$-competition graph

Let $k=0.2 e^{i 0.2 \pi}$, then there is only one edge in $\mathrm{CF}$ $0.2 e^{i 0.2 \pi}$-competition graph as

$\left|\left(\aleph^{p}(j) \cap \aleph^{p}(l)\right)\right|_{\xi}=1.36>0.2$,

$\left|\left(\aleph^{p}(j) \cap \aleph^{p}(l)\right)\right|_{\varphi}=0.8 \pi>0.2 \pi$.

The corresponding $\mathfrak{C}_{0.2 e^{i 0.2 \pi}}(\vec{\Omega})$ is shown in Fig. 4 .

Definition 14 Consider a CFG $\Omega=(W, \widehat{\mathbb{P}}, \widehat{\mathbb{Q}})$. If $\forall g, f \in$ W

$\xi_{\widehat{\mathbb{Q}}}(g, f)>\frac{1}{2}\left(\xi_{\widehat{\mathbb{P}}}(g) \wedge \xi_{\widehat{\mathbb{P}}}(f)\right)$,

$\varphi_{\widehat{\mathbb{Q}}}(g, f)>\frac{1}{2}\left[2 \pi\left(\frac{\varphi_{\widehat{\mathbb{P}}}(g)}{2 \pi} \wedge \frac{\varphi_{\widehat{\mathbb{P}}}(f)}{2 \pi}\right)\right]$,

then the edge $(g, f)$ is said to be a strong edge.

Theorem 1 Let $\vec{\Omega}=(W, \widehat{\mathbb{P}}, \overrightarrow{\mathbb{Q}})$ be a CFDG. If there exists only one element in $C F S\left(\aleph^{p}(g) \cap \aleph^{p}(f)\right)$, and $\left|\aleph^{p}(g) \cap \aleph^{p}(f)\right|_{\xi}>\frac{1}{2}$ and $\left|\aleph^{p}(g) \cap \aleph^{p}(f)\right|_{\theta}>1 \pi$, then the edge $(g, f)$ of $\mathfrak{C}(\vec{\Omega})$ is strong.

Proof Let $\vec{\Omega}=(W, \widehat{\mathbb{P}}, \overrightarrow{\mathbb{Q}})$ be a CFDG. Let $\left(\aleph^{p}(g) \cap\right.$ $\left.\aleph^{p}(f)\right)=\left\{\left(x, c e^{i \theta}\right)\right\}$, where $c e^{i \theta}$ is the MV of the edge $(g, c)$ or $(f, c)$. Then clearly

$\hbar\left(\aleph^{p}(g) \cap \aleph^{p}(f)\right)=c e^{i \theta}=\left|\left(\aleph^{p}(g) \cap \aleph^{p}(f)\right)\right|$, where

$\hbar_{\xi}\left(\aleph^{p}(g) \cap \aleph^{p}(f)\right)=c=\left|\left(\aleph^{p}(g) \cap \aleph^{p}(f)\right)\right|_{\xi}$,

$\hbar_{\varphi}\left(\aleph^{p}(g) \cap \aleph^{p}(f)\right)=\theta=\left|\left(\aleph^{p}(g) \cap \aleph^{p}(f)\right)\right|_{\varphi}$.

So, the MV of the edge $(g, f)$ in CFCG is

$\xi_{\widehat{\mathbb{Q}}}(g, f)=\left(\xi_{\widehat{\mathbb{P}}}(g) \wedge \xi_{\widehat{\mathbb{P}}}(f)\right) \times \hbar_{\xi}\left(\aleph^{p}(g) \cap \aleph^{p}(f)\right)$,

$\xi_{\widehat{\mathbb{Q}}}(g, f)=\left(\xi_{\widehat{\mathbb{P}}}(g) \wedge \xi_{\widehat{\mathbb{P}}}(f)\right) \times c$,

$\begin{aligned} \varphi_{\widehat{\mathbb{Q}}}(g, f)= & 2 \pi\left[\left(\frac{\varphi_{\widehat{\mathbb{P}}}(g)}{2 \pi} \wedge \frac{\varphi_{\widehat{\mathbb{P}}}(f)}{2 \pi}\right)\right. \\ & \left.\times \frac{\hbar_{\varphi}\left(\aleph^{p}(g) \cap \aleph^{p}(f)\right)}{2 \pi}\right],\end{aligned}$

$\varphi_{\widehat{\mathbb{Q}}}(g, f)=2 \pi\left[\left(\frac{\varphi_{\widehat{\mathbb{P}}}(g)}{2 \pi} \wedge \frac{\varphi_{\widehat{\mathbb{P}}}(f)}{2 \pi}\right) \times \frac{\theta}{2 \pi}\right]$.

Thus, in $\mathfrak{C}(\vec{\Omega})$, the edge $(g, f)$ is strong as $c>\frac{1}{2}$ and $\theta>1 \pi$.

Definition 15 Let $B=\left\{\left(q, \xi_{B}(q) e^{i \varphi_{B}(q)} \mid q \in Z\right)\right\}$, be a CFS. The the support of CFS is a subset $B_{0}$ of $Z$, defined as

$B_{0}=\left\{q \in Z: \xi_{B}(q) \neq 0\right\}$

Now, we discuss $p$-competition complex fuzzy graph.

Definition 16 Let $\breve{p}$ be positive integer. The $\breve{p}$-competition $\operatorname{CFG} \mathfrak{C}^{\breve{p}}(\vec{\Omega})$ of a CFDG $\vec{\Omega}=(W, \widehat{\mathbb{P}}, \overrightarrow{\mathbb{Q}})$ is an undirected CFG $\Omega=(W, \widehat{\mathbb{P}}, \widehat{\mathbb{Q}})$ with same $\mathrm{CF}$ node set as $\vec{\Omega}$ and possesses a $\mathrm{CF}$ edge joining two distinct nodes $g$ and $f \in W$ in $\mathfrak{C}^{\breve{p}}(\vec{\Omega})$ if and only if $\left|\operatorname{supp}\left(\aleph^{p}(g) \cap \aleph^{p}(f)\right)\right| \geq \breve{p}$. The MV of edge $(g, f) \in \mathfrak{C}^{\breve{p}}(\vec{\Omega})$ is calculated as

$$
\begin{aligned}
\xi_{\widehat{\mathbb{Q}}}(g, f)= & \left(\frac{(t-\breve{p})+1}{t}\right)\left(\xi_{\widehat{\mathbb{P}}}(g) \wedge \xi_{\widehat{\mathbb{P}}}(f)\right) \\
& \times \hbar_{\xi}\left(\aleph^{p}(g) \cap \aleph^{p}(f)\right), \\
\varphi_{\widehat{\mathbb{Q}}}(g, f)= & 2 \pi\left[\left(\frac{(t-\breve{p})+1}{t}\right)\left(\frac{\varphi_{\breve{\mathbb{P}}}(g)}{2 \pi} \wedge \frac{\varphi_{\widehat{\mathbb{P}}}(f)}{2 \pi}\right)\right. \\
& \left.\times \frac{\hbar_{\varphi}\left(\aleph^{p}(g) \cap \aleph^{p}(f)\right)}{2 \pi}\right],
\end{aligned}
$$


Fig. 5 Two-competition complex fuzzy graph

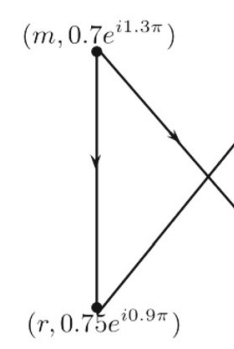

(a) CFDG $(\vec{\Omega})$

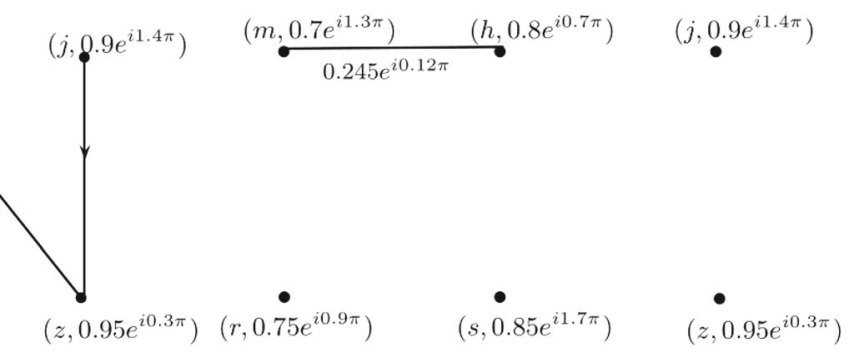

(b) $C^{2}(\vec{\Omega})$
Table 6 CFON of the nodes

\begin{tabular}{ll}
\hline $\mathrm{g}$ & $\aleph^{p}(g)$ \\
\hline $\mathrm{m}$ & $\left\{\left(r, 0.7 e^{i 0.8 \pi}\right),\left(s, 0.7 e^{i 1.3 \pi}\right)\right\}$ \\
$\mathrm{h}$ & $\left\{\left(r, 0.6 e^{i 0.3 \pi}\right),\left(s, 0.8 e^{i 0.7 \pi}\right),\left(z, 0.8 e^{i 0.3 \pi}\right)\right\}$ \\
$\mathrm{j}$ & $\left\{\left(z, 0.8 e^{i 0.3 \pi}\right)\right\}$ \\
$\mathrm{r}$ & $\varnothing$ \\
$\mathrm{s}$ & $\varnothing$ \\
$\mathrm{z}$ & $\varnothing$ \\
\hline
\end{tabular}

where $t=\left|\operatorname{supp}\left(\aleph^{p}(g) \cap \aleph^{p}(f)\right)\right|$.

Example 4 Let $\vec{\Omega}=(W, \widehat{\mathbb{P}}, \overrightarrow{\mathbb{Q}})$ be a CFDG as presented in Fig. 5a, given by

$$
\begin{aligned}
\widehat{\mathbb{P}} & =\left\langle\frac{m}{0.7 e^{i 1.3 \pi}}, \frac{h}{0.8 e^{i 0.7 \pi}}, \frac{j}{0.9 e^{i 1.4 \pi}}, \frac{r}{0.75 e^{i 0.9 \pi}},\right. \\
\frac{s}{0.85 e^{i 1.7 \pi}}, & \left.\frac{z}{0.95 e^{i 0.3 \pi}}\right\rangle, \\
\overrightarrow{\mathbb{Q}} & =\left\langle\frac{(m, r)}{0.7 e^{i 0.8 \pi}}, \frac{(m, s)}{0.7 e^{i 1.3 \pi}}, \frac{\overrightarrow{(h, r)}}{0.6 e^{i 0.3 \pi}}, \frac{\overrightarrow{(h, s)}}{0.8 e^{i 0.7 \pi}},\right. \\
\overrightarrow{(h, z)} & \left.\frac{(j, z)}{0.8 e^{i 0.3 \pi}}, \frac{}{0.9 e^{i 0.3 \pi}}\right\rangle .
\end{aligned}
$$

The CFON of the nodes are give in Table 6.

The corresponding two-competition CFG has only one edge as

$\aleph^{p}(m) \cap \aleph^{p}(h)=\left\{\left(r, 0.6 e^{i 0.3 \pi}\right),\left(s, 0.7 e^{i 0.7 \pi}\right)\right\} \neq \varnothing$.

The corresponding $\mathfrak{C}^{2}(\vec{\Omega})$ is shown in Fig. $5 b$.

Theorem 2 Let $\vec{\Omega}=(W, \widehat{\mathbb{P}}, \overrightarrow{\mathbb{Q}})$ be a CFDG. Then the edge $(g, f)$ is strong, if $\hbar_{\xi}\left(\aleph^{p}(g) \cap \aleph^{p}(f)\right)=1$ and $\hbar_{\varphi}\left(\aleph^{p}(g) \cap \aleph^{p}(f)\right)=2 \pi$ in $\mathfrak{C}^{\left[\frac{t}{2}\right]}(\vec{\Omega})$, where $t=$ $\left|\operatorname{supp}\left(\aleph^{p}(g) \cap \aleph^{p}(f)\right)\right|$.

Proof Consider $\vec{\Omega}=(W, \widehat{\mathbb{P}}, \overrightarrow{\mathbb{Q}})$ is a CFDG. Let $\Omega=$ $(W, \widehat{\mathbb{P}}, \widehat{\mathbb{Q}})$ be the corresponding $\left[\frac{t}{2}\right]-\mathrm{CFCG}$, where $t=$ $\left|\operatorname{supp}\left(\aleph^{p}(g) \cap \aleph^{p}(f)\right)\right|$. According to the statement, we have $\hbar_{\xi}\left(\aleph^{p}(g) \cap \aleph^{p}(f)\right)=1$ and $\hbar_{\varphi}\left(\aleph^{p}(g) \cap \aleph^{p}(f)\right)=2 \pi$. Then by Definition 16 , we have

$$
\begin{aligned}
\xi_{\widehat{\mathbb{Q}}}(g, f)= & \frac{t-\left[\frac{t}{2}\right]+1}{t}\left(\xi_{\widehat{\mathbb{P}}}(g) \wedge \xi_{\widehat{\mathbb{P}}}(f)\right) \\
& \times \hbar_{\xi}\left(\aleph^{p}(g) \cap \aleph^{p}(f)\right) \\
= & \frac{t-\left[\frac{t}{2}\right]+1}{t}\left(\xi_{\widehat{\mathbb{P}}}(g) \wedge \xi_{\widehat{\mathbb{P}}}(f)\right), \\
\varphi_{\widehat{\mathbb{Q}}}(g, f)= & 2 \pi\left[\frac{t-\left[\frac{t}{2}\right]+1}{t}\left(\frac{\varphi_{\widehat{\mathbb{P}}}(g)}{2 \pi} \wedge \frac{\varphi_{\widehat{\mathbb{P}}}(f)}{2 \pi}\right)\right. \\
& \left.\times \frac{\hbar_{\varphi}\left(\aleph^{p}(g) \cap \aleph^{p}(f)\right)}{2 \pi}\right] \\
= & 2 \pi\left[\frac{t-\left[\frac{t}{2}\right]+1}{t}\left(\frac{\varphi_{\widehat{\mathbb{P}}}(g)}{2 \pi} \wedge \frac{\varphi_{\widehat{\mathbb{P}}}(f)}{2 \pi}\right) \times \frac{2 \pi}{2 \pi}\right] .
\end{aligned}
$$

Thus, $\xi_{\widehat{\mathbb{Q}}}(g, f)>\frac{1}{2}\left(\xi_{\widehat{\mathbb{P}}}(g) \wedge \xi_{\widehat{\mathbb{P}}}(f)\right)$ and $\varphi_{\widehat{\mathbb{Q}}}(g, f)>$ $\frac{1}{2}\left[2 \pi\left(\frac{\varphi_{\widehat{\mathbb{P}}}(g)}{2 \pi} \wedge \frac{\varphi_{\widehat{\mathbb{P}}}(f)}{2 \pi}\right)\right]$, as $\frac{t-\left[\frac{t}{2}\right]+1}{t}>\frac{1}{2}$. Hence, the edge $(g, f)$ is strong.

\section{Complex fuzzy set applied to neighborhood graphs}

Now, we discuss complex fuzzy neighborhood graphs by the combination of CFS and neighborhood graphs. Before the introduction of CF neighborhood graphs (CFNGs), we first define complex fuzzy open and closed neighborhood of a node in CFGs below.

Definition 17 For a node $g$ of a $\operatorname{CFG} \Omega=(W, \widehat{\mathbb{P}}, \widehat{\mathbb{Q}})$ the $\mathrm{CF}$ open neighborhood $\left(\mathrm{CFO}^{p} N\right)$ is a CFS

$\aleph(g)=\left(S_{g}, m_{g} e^{i \eta_{g}}\right)$,

where

$S_{g}=\left\{f \mid \xi_{\widehat{Q}}(g, f)>0\right\}$ 
such that $m_{g}: S_{g} \rightarrow[0,1]$ given by $m_{g}(f)=\xi_{\widehat{\mathbb{Q}}}(g, f)$ and $\eta_{g}: S_{g} \rightarrow[0,2 \pi]$ given by $\eta_{g}(f)=\varphi_{\widehat{Q}}(g, f)$.

For each node $g \in W$, CF singleton set is given as

$\widehat{\mathbb{P}}_{g}=\left(\{g\}, \xi_{\widehat{\mathbb{P}}}^{\prime} e^{i \varphi_{\widehat{\mathbb{P}}}^{\prime}}\right)$,

where $\xi_{\widehat{\mathbb{P}}}^{\prime}:\{g\} \rightarrow[0,1]$ and $\varphi_{\widehat{\mathbb{P}}}^{\prime}:\{g\} \rightarrow[0,2 \pi]$ given by $\xi_{\widehat{\mathbb{P}}}^{\prime}(g)=\xi_{\widehat{\mathbb{P}}}(g)$ and $\varphi_{\widehat{\mathbb{P}}}^{\prime}(g)=\varphi_{\widehat{\mathbb{P}}}(g)$, respectively. Now, we define $\mathrm{CF}$ closed neighborhood $\left(C F C^{l} N\right)$ of a node $g$ as

$$
\aleph[g]=\aleph(g) \cup \widehat{\mathbb{P}}_{g}
$$

Next, we define $\mathrm{CF}$ open neighborhood graphs and CF closed neighborhood graphs. Then on the bases of these CFGs, we define complex fuzzy $k$-neighborhood graphs of both close and open types.

Definition 18 Let $\Omega=(W, \widehat{\mathbb{P}}, \widehat{\mathbb{Q}})$ be a CFG. Complex fuzzy open neighborhood graph $\left(C F O^{p} N G\right)$ of $\Omega$ is a CFG denoted by $\mathfrak{N}(\Omega)=\left(W, \widehat{\mathbb{P}}, \widehat{\mathbb{Q}}^{\prime}\right)$ with same $\mathrm{CF}$ node set as $\Omega$ and possesses a CF edge joining two distinct nodes $g$ and $f$ in $\mathfrak{N}(\Omega)$ if and only if the CFS $\aleph(g) \cap \aleph(f) \neq \varnothing$ in $\Omega$ and the MV of the edge $(g, f)$ in $\mathfrak{N}(\Omega)$ is calculated as

$\xi_{\widehat{\mathbb{Q}}^{\prime}}(g, f)=\left(\xi_{\widehat{\mathbb{P}}}(g) \wedge \xi_{\widehat{\mathbb{P}}}(f)\right) \times \hbar_{\xi}(\aleph(g) \cap \aleph(f))$,

$\varphi_{\widehat{\mathbb{Q}}^{\prime}}(g, f)=2 \pi\left[\left(\frac{\varphi_{\widehat{\mathbb{P}}}(g)}{2 \pi} \wedge \frac{\varphi_{\widehat{\mathbb{P}}}(f)}{2 \pi}\right) \times \frac{\hbar_{\varphi}(\aleph(g) \cap \aleph(f))}{2 \pi}\right]$.

Definition 19 Let $\Omega=(W, \widehat{\mathbb{P}}, \widehat{\mathbb{Q}})$ be a CFG. Complex fuzzy closed neighborhood graph $\left(C F C^{l} N G\right)$ of $\Omega$ is a CFG denoted by $\mathfrak{N}[\Omega]=\left(W, \widehat{\mathbb{P}}, \widehat{\mathbb{Q}}^{\prime}\right)$ with same $\mathrm{CF}$ node set as $\Omega$ and possesses a CF edge joining two distinct nodes $g$ and $f \in W$ in $\mathfrak{N}[\Omega]$ if and only if the CFS $\aleph[g] \cap \aleph[f] \neq \varnothing$ in $\Omega$ and the $\mathrm{MV}$ of the edge $(g, f)$ in $\mathfrak{N}[\Omega]$ is calculated as

$\xi_{\widehat{\mathbb{Q}}^{\prime}}(g, f)=\left(\xi_{\widehat{\mathbb{P}}}(g) \wedge \xi_{\widehat{\mathbb{P}}}(f)\right) \times \hbar_{\xi}(\aleph[g] \cap \aleph[f])$,

$\varphi_{\widehat{\mathbb{Q}}^{\prime}}(g, f)=2 \pi\left[\left(\frac{\varphi_{\widehat{\mathbb{P}}}(g)}{2 \pi} \wedge \frac{\varphi_{\widehat{\mathbb{P}}}(f)}{2 \pi}\right) \times \frac{\hbar_{\varphi}(\aleph[g] \cap \aleph[f])}{2 \pi}\right]$.

Definition 20 Complex fuzzy $(k)$-neighborhood graph of a CFG $\Omega=(W, \widehat{\mathbb{P}}, \widehat{\mathbb{Q}})$ is a CFG $\mathfrak{N}_{k}(\Omega)=\left(W, \widehat{\mathbb{P}}, \widehat{\mathbb{Q}}^{\prime}\right)$ with same node set as $\Omega$ and possesses a CF edge joining two distinct nodes $g$ and $f$ in $\mathfrak{N}_{k}(\Omega)$ if and only if $x^{\prime}>r$ and $\omega^{\prime}>\theta$ and the MV of the edge $(g, f)$ in $\mathfrak{N}_{k}(\Omega)$ is calculated as

$$
\begin{aligned}
\xi_{\widehat{\mathbb{Q}}^{\prime}}(g, f)= & \left(\frac{x^{\prime}-r}{x^{\prime}}\right)\left(\xi_{\widehat{\mathbb{P}}}(g) \wedge \xi_{\widehat{\mathbb{P}}}(f)\right) \\
& \times \hbar_{\xi}(\aleph(g) \cap \aleph(f)), \\
\varphi_{\widehat{\mathbb{Q}}^{\prime}}(g, f)= & 2 \pi\left[\frac{\omega^{\prime}-\theta}{\omega^{\prime}}\left(\frac{\varphi_{\widehat{\mathbb{P}}}(g)}{2 \pi} \wedge \frac{\varphi_{\widehat{\mathbb{P}}}(f)}{2 \pi}\right)\right. \\
& \left.\times \frac{\hbar_{\varphi}(\aleph(g) \cap \aleph(f))}{2 \pi}\right] .
\end{aligned}
$$

Here, $k$ is a complex number given by $k=r e^{i \theta}$ and $\mid \aleph(g) \cap$ $\aleph(f) \mid=x^{\prime} e^{i \omega^{\prime}}$.

Definition 21 Let $\Omega=(W, \widehat{\mathbb{P}}, \widehat{\mathbb{Q}})$ be a CFG. Complex fuzzy $[k]$-neighborhood graph of $\Omega=(W, \widehat{\mathbb{P}}, \widehat{\mathbb{Q}})$ is a $\mathrm{CFG}$ $\mathfrak{N}_{k}[\Omega]=\left(W, \widehat{\mathbb{P}}, \widehat{\mathbb{Q}^{\prime}}\right)$ with same node set as $\Omega$ and possesses a CF edge joining two distinct nodes $g$ and $f$ in $\mathfrak{N}_{k}[\Omega]$ if and only if $x^{\prime \prime}>r$ and $\omega^{\prime \prime}>\theta$ in $\Omega$ and the MV of the edge $(g, f)$ in $\mathfrak{N}_{k}[\Omega]$ is calculated as

$$
\begin{aligned}
\xi_{\widehat{\mathbb{Q}}^{\prime}}(g, f)= & \left(\frac{x^{\prime \prime}-r}{x^{\prime \prime}}\right)\left(\xi_{\widehat{\mathbb{P}}}(g) \wedge \xi_{\widehat{\mathbb{P}}}(f)\right) \\
& \times \hbar_{\xi}(\aleph[g] \cap \aleph[f]), \\
\varphi_{\widehat{\mathbb{Q}}^{\prime}}(g, f)= & 2 \pi\left[\frac{\omega^{\prime \prime}-\theta}{\omega^{\prime \prime}}\left(\frac{\varphi_{\widehat{\mathbb{P}}}(g)}{2 \pi} \wedge \frac{\varphi_{\widehat{\mathbb{P}}}(f)}{2 \pi}\right)\right. \\
& \left.\times \frac{\hbar_{\varphi}(\aleph[g] \cap \aleph[f])}{2 \pi}\right] .
\end{aligned}
$$

Here, $k$ is a complex number given by $k=r e^{i \theta}$ and $\mid \aleph[g] \cap$ $\aleph[f] \mid=x^{\prime \prime} e^{i \omega^{\prime \prime}}$.

Example 5 Let $\Omega=(W, \widehat{\mathbb{P}}, \widehat{\mathbb{Q}})$ be a CFG, where

$$
\begin{aligned}
& \widehat{\mathbb{P}}=\left\langle\frac{p}{0.3 e^{i 1.9 \pi}}, \frac{q}{0.6 e^{i 1.8 \pi}}, \frac{r}{0.4 e^{i 1.5 \pi}}, \frac{s}{0.5 e^{i 1.7 \pi}},\right. \\
& \left.\frac{t}{0.4 e^{i 1.9 \pi}}\right\rangle, \\
& \widehat{\mathbb{Q}}=\left\langle\frac{(p, q)}{0.3 e^{i 1.7 \pi}}, \frac{(q, r)}{0.4 e^{i 1.5 \pi}}, \frac{(r, s)}{0.4 e^{i 1.4 \pi}}, \frac{(s, t)}{0.4 e^{i 1.7 \pi}},\right. \\
& \left.\frac{(t, p)}{0.3 e^{i 1.8 \pi}}, \frac{(p, s)}{0.3 e^{i 1.7 \pi}}\right\rangle .
\end{aligned}
$$

The CFDG is displayed in Fig. 6a. The CF neighborhoods of the nodes are presented in Table 7.

The height and cardinality of CFSs $(\aleph(g) \cap \aleph(f))$ and $(\aleph[g] \cap \aleph[f])$ are displayed in Table 8.

The corresponding $\left(C F O^{p} N G\right) \mathfrak{N}(\Omega)$, the $\left(C F C^{l} N G\right)$ $\mathfrak{N}[\Omega]$, the complex fuzzy $\left(0.2 e^{i 0.2 \pi}\right)$-neighborhood graph $\mathfrak{N}_{0.2 e^{i 0.2 \pi}}(\Omega)$ and the complex fuzzy $\left[0.2 e^{i 0.2 \pi}\right]$-neighbourhood graph $\mathfrak{N}_{0.2 e^{i 0.2 \pi}}[\Omega]$ of the CFG $\Omega$ are shown in Fig. 6 .

Theorem 3 For each edge of $C F G G$, there exists one edge in $\mathfrak{N}[\Omega]$.

Proof Let $\Omega=(W, \widehat{\mathbb{P}}, \widehat{\mathbb{Q}})$ be a CFG and $(g, f)$ be an edge of CFG. Let the corresponding $\left(C F C^{l} N G\right)$ be $\mathfrak{N}[\Omega]=$ 


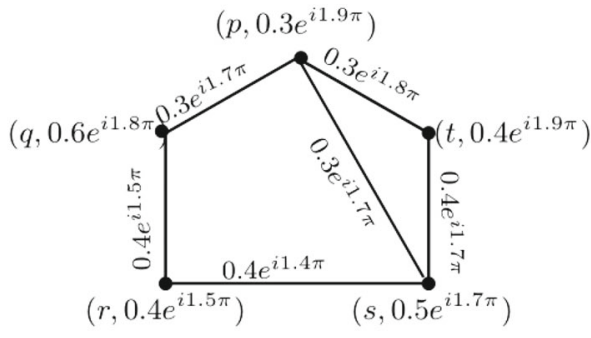

(a) $\Omega$

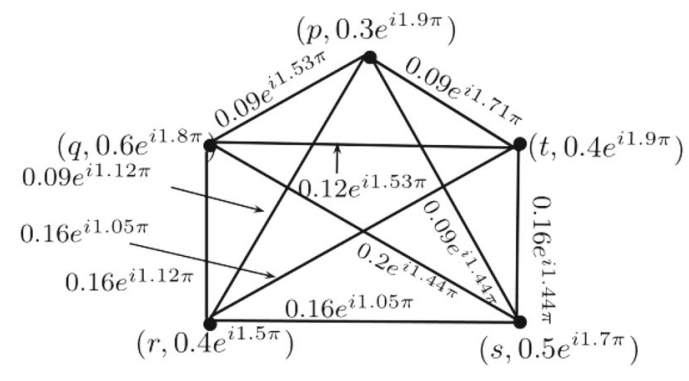

(c) $N[\Omega]$

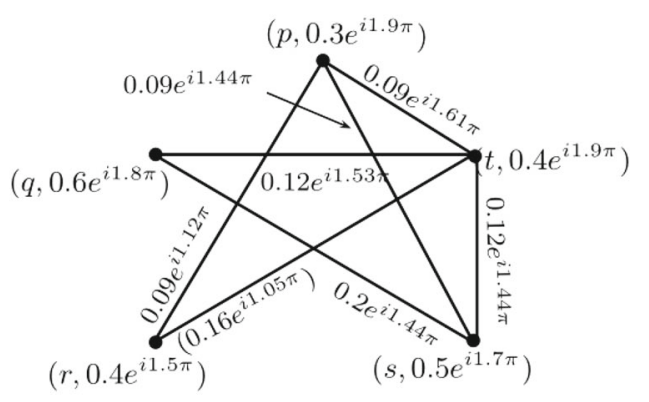

(b) $N(\Omega)$

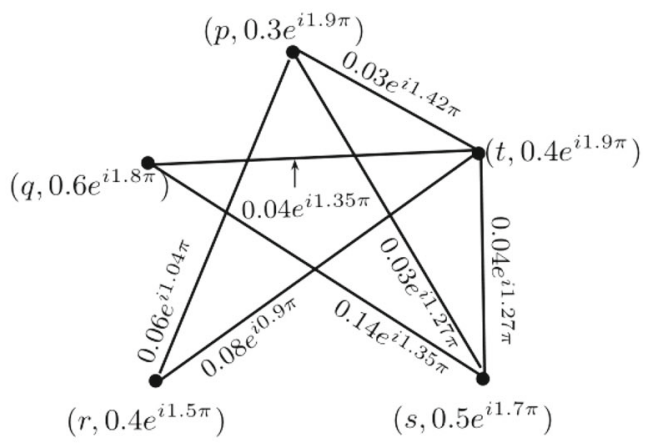

(d) $N_{0.2 e^{i 0.2 \pi}(\Omega)}$

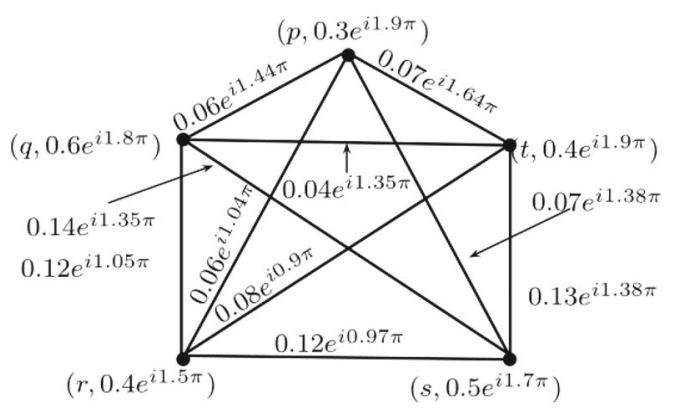

(e) $N_{0.2 e^{i 0.2 \pi}}[\Omega]$

Fig. 6 Complex fuzzy neighborhood graphs

Table $7 C F O^{p} N$ and $C F C^{l} N$ of the nodes

\begin{tabular}{lll}
\hline $\mathrm{g}$ & $\aleph(g)$ & $\aleph[g]$ \\
\hline $\mathrm{p}$ & $\left\{\left(q, 0.3 e^{i 1.7 \pi}\right)\left(t, 0.3 e^{i 1.8 \pi}\right),\left(s, 0.3 e^{i 1.7 \pi}\right)\right\}$ & $\left\{\left(q, 0.3 e^{i 1.7 \pi}\right)\left(\left(t, 0.3 e^{i 1.8 \pi}\right),\left(s, 0.3 e^{i 1.7 \pi}\right)\right)\right\}$ \\
$\mathrm{q}$ & & $\cup\left\{\left(p, 0.3 e^{i 1.9 \pi}\right)\right\}$ \\
$\mathrm{r}$ & $\left\{\left(p, 0.3 e^{i 1.7 \pi}\right),\left(r, 0.4 e^{i 1.5 \pi}\right)\right\}$ & $\left\{\left(p, 0.3 e^{i 1.7 \pi}\right),\left(r, 0.4 e^{i 1.5 \pi}\right)\right\} \cup\left\{\left(q, 0.6 e^{i 1.8 \pi}\right)\right\}$ \\
$\mathrm{s}$ & $\left\{\left(q, 0.4 e^{i 1.5 \pi}\right),\left(s, 0.4 e^{i 1.4 \pi}\right)\right\}$ & $\left\{\left(q, 0.4 e^{i 1.5 \pi}\right),\left(s, 0.4 e^{i 1.4 \pi}\right)\right\} \cup\left\{\left(r, 0.4 e^{i 1.5 \pi}\right)\right\}$ \\
& $\left\{\left(r, 0.4 e^{i 1.4 \pi}\right),\left(t, 0.4 e^{i 1.7 \pi}\right),\left(p, 0.3 e^{i 1.7 \pi}\right)\right\}$ & $\left\{\left(r, 0.4 e^{i 1.4 \pi}\right),\left(t, 0.4 e^{i 1.7 \pi}\right),\left(p, 0.3 e^{i 1.7 \pi}\right)\right\}$ \\
$\mathrm{t}$ & & $\cup\left\{\left(s, 0.5 e^{i 1.7 \pi}\right)\right\}$ \\
\hline
\end{tabular}


Table 8 Height and cardinality of CFSs $(\aleph(g) \cap \aleph(f))$ and $(\aleph[g] \cap \aleph[f])$

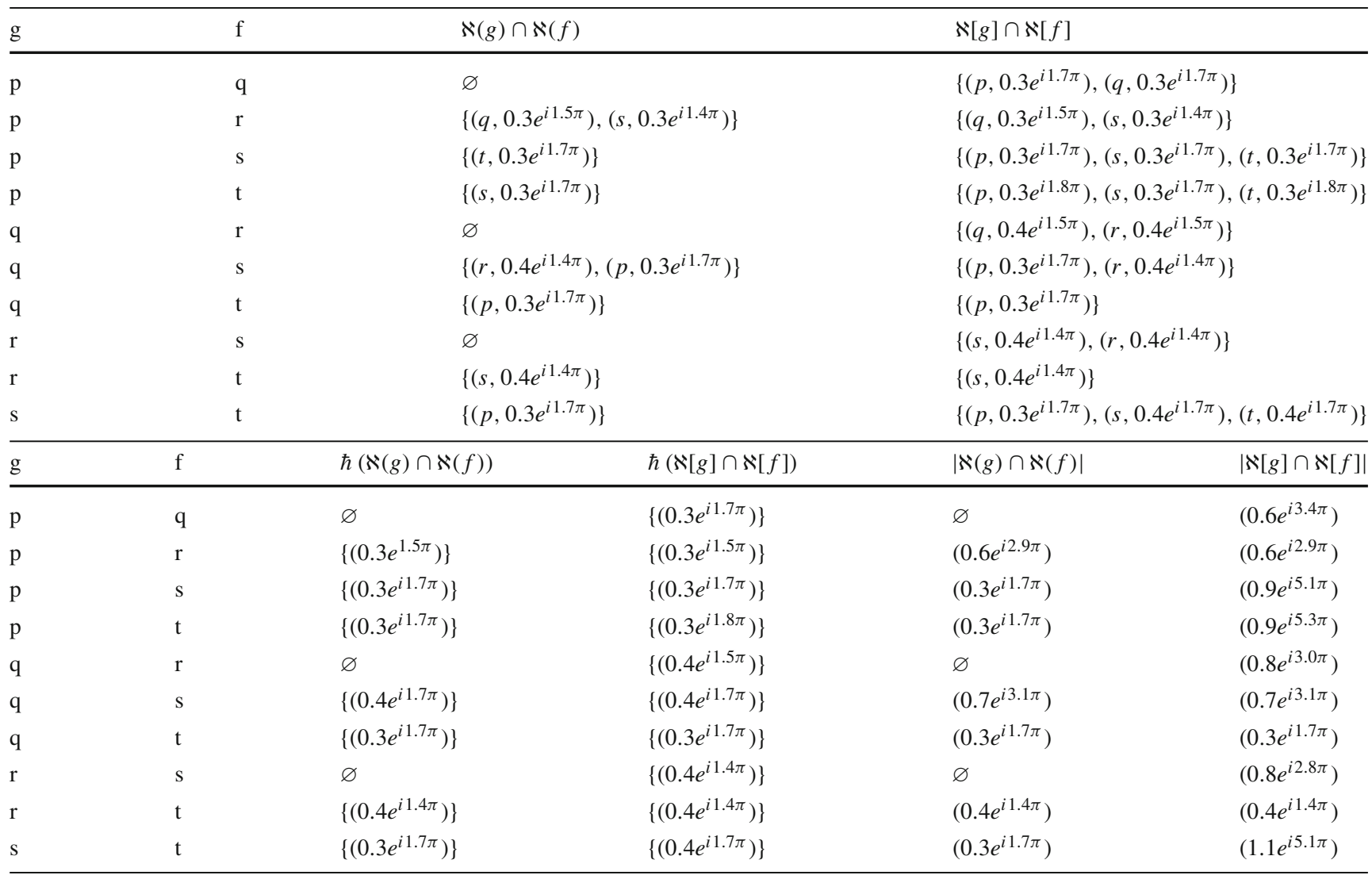

$\left(W, \widehat{\mathbb{P}}, \widehat{\mathbb{Q}}^{\prime}\right)$. Then $g, f \in \aleph[g]$ and $g, f \in \aleph[f]$. Thus, $g, f \in \aleph[g] \cap \aleph[f]$. So, $\hbar_{\xi}(\aleph[g] \cap \aleph[f]) \neq 0$, and $\hbar_{\varphi}(\aleph[g] \cap \aleph[f]) \neq 0$. Thus, the amplitude and phase terms of the MV are

$\xi_{\widehat{\mathbb{Q}}^{\prime}}(g, f)=\left(\xi_{\widehat{\mathbb{P}}}(g) \wedge \xi_{\widehat{\mathbb{P}}}(f)\right) \times \hbar_{\xi}(\aleph[g] \cap \aleph[f]) \neq 0$,

$\varphi_{\widehat{\mathbb{Q}}^{\prime}}(g, f)=2 \pi\left[\left(\frac{\varphi_{\widehat{\mathbb{P}}}(g)}{2 \pi} \wedge \frac{\varphi_{\widehat{\mathbb{P}}}(f)}{2 \pi}\right) \times \frac{\hbar_{\varphi}(\aleph[g] \cap \aleph[f])}{2 \pi}\right] \neq 0$.

Thus, for each edge $(g, f)$ in $\Omega$ there exists an edge $(g, f)$ in $\mathfrak{N}[\Omega]$.

Definition 22 Let $\vec{\Omega}=(W, \widehat{\mathbb{P}}, \overrightarrow{\mathbb{Q}})$ be a CFDG. The underlying CFG of $\vec{\Omega}$ is the CFG symbolized by $\mathfrak{U}(\Omega)$ and is defined as $\mathfrak{U}(\Omega)=(W, \widehat{\mathbb{P}}, \widehat{\mathbb{Q}})$, where $\widehat{\mathbb{P}}$ and $\widehat{\mathbb{Q}}$ are CFS and CFR on $W$ such that

$\xi_{\widehat{\mathbb{Q}}}(g, f)=\left(\xi_{\widehat{\mathbb{P}}} \overrightarrow{(g, f)} \wedge \xi_{\widehat{\mathbb{P}}} \overrightarrow{(f, g)}\right)$,

$\varphi_{\widehat{\mathbb{Q}}}(g, f)=\left(\varphi_{\widehat{\mathbb{P}}} \overrightarrow{(g, f)} \wedge \varphi_{\widehat{\mathbb{P}}} \overrightarrow{(f, g)}\right)$,

for all $g, f \in W$.
Now, we establish a relation between $\mathfrak{N}_{k}(\Omega)$ and $\mathfrak{C}_{k}(\Omega)$. Theorem 4 If a symmetric CFDG $\vec{\Omega}$ is loopless, then $\mathfrak{C}_{k}(\vec{\Omega})=\mathfrak{N}_{k}(\mathfrak{U}(\Omega))$, where $\mathfrak{U}(\Omega)$ is the underlying $C F G$ of $\vec{\Omega}$. (Here, $\left.k=r e^{i \theta}\right)$

Proof Let $\vec{\Omega}=(W, \widehat{\mathbb{P}}, \overrightarrow{\mathbb{Q}})$ be a CFDG and the corresponding underlying CFG of $\vec{\Omega}$ be $\mathfrak{U}(\Omega)=(W, \widehat{\mathbb{P}}, \widehat{\mathbb{Q}})$. Also, let $\mathfrak{C}_{k}(\vec{\Omega})=(W, \widehat{\mathbb{P}}, \breve{\mathbb{C}})$ and $\mathfrak{N}_{k}(\mathfrak{U}(\Omega))=$ $\left(W, \widehat{\mathbb{P}}, \breve{C}^{\prime}\right)$. The complex fuzzy node of $\mathfrak{C}_{k}(\Omega)$ is same as $\vec{\Omega}$. Also the node set of an underlying CFG is same as the complex fuzzy node of CFDG. Hence, $\mathfrak{N}_{k}(\mathfrak{U}(\Omega))$ has the same complex fuzzy node set as $\mathfrak{C}_{k}(\Omega)$. Now, we need to show that complex fuzzy edge set of $\mathfrak{C}_{k}(\vec{\Omega})$ is equal to the edge set of $\mathfrak{N}_{k}(\mathfrak{U}(\Omega))$. For this, we need to show that $\xi_{\breve{C}}(g, f)=\xi_{\breve{C}^{\prime}}(g, f)$ and $\varphi_{\breve{C}}(g, f)=\varphi_{\breve{C}^{\prime}}(g, f)$, for all $g, f \in W$.

Case 1 If $\left|\left(\aleph^{p}(g) \cap \aleph^{p}(f)\right)\right|_{\xi}=r^{\prime} \leq r$ and $\mid\left(\aleph^{p}(g) \cap \aleph^{p}\right.$ $(f))\left.\right|_{\varphi}=\theta^{\prime} \leq \theta$ in $\vec{\Omega}$, then $\xi_{\breve{\mathbb{C}}}(g, f)=$ 0 and $\varphi_{\breve{C}}(g, f)=0$ in $\mathfrak{C}_{k}(\vec{\Omega})$. As $\vec{\Omega}$ is symmetric CFG, $|(\aleph(g) \cap \aleph(f))|_{\xi}=x^{\prime} \leq r$ 
and $|(\aleph(g) \cap \aleph(f))|_{\varphi}=\omega^{\prime} \leq \theta$ in $\mathfrak{U}(\Omega)$. So, $\xi_{\breve{C}^{\prime}}(g, f)=0$ and $\varphi_{\breve{C}^{\prime}}(g, f)=0$ in $\mathfrak{N}_{k}(\mathfrak{U}(\Omega))$.

Case 2 If $r^{\prime}>r$ and $\theta^{\prime}>\theta$ in $\vec{\Omega}$, then $\xi_{\breve{C}}(g, f)>0$ and $\varphi_{\breve{C}}(g, f)>0$ in $\mathfrak{C}_{k}(\vec{\Omega})$. Then amplitude and phase term in $\mathfrak{C}_{k}(\vec{\Omega})$ are given by

$$
\begin{aligned}
\xi_{\breve{\mathbb{C}}}(g, f)= & \left(\frac{\left(r^{\prime}-r\right)}{r^{\prime}}\right)\left(\xi_{\widehat{\mathbb{P}}}(g) \wedge \xi_{\widehat{\mathbb{P}}}(f)\right) \\
& \times \hbar_{\xi}\left(\aleph^{p}(g) \cap \aleph^{p}(f)\right), \\
\varphi_{\breve{\mathbb{C}}}(g, f)= & 2 \pi\left[\frac{\theta^{\prime}-\theta}{\theta^{\prime}}\left(\frac{\varphi_{\widehat{\mathbb{P}}}(g)}{2 \pi} \wedge \frac{\varphi_{\widehat{\mathbb{P}}}(f)}{2 \pi}\right)\right. \\
& \left.\times \frac{\hbar_{\varphi}\left(\aleph^{p}(g) \cap \aleph^{p}(f)\right)}{2 \pi}\right] .
\end{aligned}
$$

As $\vec{\Omega}$ is symmetric CFDG, $x^{\prime}>r$ and $\omega^{\prime}>\theta$ in $\mathfrak{U}(\Omega)$. The amplitude and phase term of $\mathrm{MV}$ in $\mathfrak{N}_{k}(\mathfrak{U}(\Omega))$ are

$$
\begin{aligned}
\xi_{\breve{\mathbb{C}}^{\prime}}(g, f)= & \left(\frac{\left(x^{\prime}-r\right)}{x^{\prime}}\right)\left(\xi_{\widehat{\mathbb{P}}}(g) \wedge \xi_{\widehat{\mathbb{P}}}(f)\right) \\
& \times \hbar_{\xi}(\aleph(g) \cap \aleph(f)), \\
\varphi_{\breve{C}^{\prime}}(g, f)= & 2 \pi\left[\frac{\left(\omega^{\prime}-\theta\right)}{\omega^{\prime}}\left(\frac{\varphi_{\widehat{\mathbb{P}}}(g)}{2 \pi} \wedge \frac{\varphi_{\widehat{\mathbb{P}}}(f)}{2 \pi}\right)\right. \\
& \left.\times \frac{\hbar_{\varphi}(\aleph(g) \cap \aleph(f))}{2 \pi}\right] .
\end{aligned}
$$

As $\vec{\Omega}$ is symmetric then it is clear that $\hbar\left(\aleph^{p}(g) \cap \aleph^{p}(f)\right)$ in $\vec{\Omega}$ is equal to $\hbar(\aleph(g) \cap \aleph(f))$ in $\mathfrak{U}(\vec{\Omega})$, i.e.,

$\hbar_{\xi}\left(\aleph^{p}(g) \cap \aleph^{p}(f)\right)=\hbar_{\xi}(\aleph(g) \cap \aleph(f))$,

$\hbar_{\varphi}\left(\aleph^{p}(g) \cap \aleph^{p}(f)\right)=\hbar_{\varphi}(\aleph(g) \cap \aleph(f))$.

Similarly, $\left|\aleph^{p}(g) \cap \aleph^{p}(f)\right|$ in $\vec{\Omega}$ is equal to $|(\aleph(g) \cap \aleph(f))|$ in $\mathfrak{U}(\vec{\Omega})$, i.e.,

$$
\begin{gathered}
\left|\left(\aleph^{p}(g) \cap \aleph^{p}(f)\right)\right|_{\xi}=r^{\prime}=x^{\prime}=|(\aleph(g) \cap \aleph(f))|_{\xi}, \\
\left|\left(\aleph^{p}(g) \cap \aleph^{p}(f)\right)\right|_{\varphi}=\theta^{\prime}=\omega^{\prime}=|(\aleph(g) \cap \aleph(f))|_{\varphi} .
\end{gathered}
$$

Hence, $\xi_{\breve{\mathbb{C}}}(g, f)=\xi_{\breve{\mathbb{C}}^{\prime}}(g, f)$ and $\varphi_{\breve{\mathbb{C}}}(g, f)=\varphi_{\breve{\mathbb{C}}^{\prime}}(g, f)$ for all $g, f \in W$.

Theorem 5 If a symmetric $C F G \vec{\Omega}$ has a loop at every node, then $\mathfrak{C}_{k}(\vec{\Omega})=\mathfrak{N}_{k}[\mathfrak{U}(\Omega)]$, where $\mathfrak{U}(\Omega)$ is the loopless underlying $C F G$ of $\vec{\Omega}$.

Proof Similar to Theorem 4.

\section{Complex fuzzy set applied to $\boldsymbol{m}$-step competition graphs}

Now, we define $m$-step CFCG by combining CFS with $m$-step CGs. Before defining $m$-step CFCG, we first discuss $m$-step complex fuzzy digraph ( $m$-step CFDG), complex fuzzy $m$ step out-neighborhood of a node and then complex fuzzy $m$-step in-neighborhood of a node below.

Definition 23 Let $\vec{\Omega}=(W, \widehat{\mathbb{P}}, \overrightarrow{\mathbb{Q}})$ be CFDG. Then $m$ step CFDG of $\vec{\Omega}$ is symbolized by $\vec{\Omega}_{m}=(\vec{\Omega}, A, \vec{B})$ with same CF node set as $\vec{\Omega}$ and has a CF edge connecting two vertices $g$ and $f$ in $\vec{\Omega}_{m}$ if it has a CF directed path (CFDP) of length $m$ from $g$ to $f$, i.e., $\vec{P}_{(g, f)}^{m}$ in $\vec{\Omega}$.

Definition 24 For a node $g$ of a $\mathrm{CFG} \vec{\Omega}=(W, \widehat{\mathbb{P}}, \overrightarrow{\mathbb{Q}})$, the CF $m$-step out-neighborhood is CFS

$\aleph_{m}^{p}(g)=\left(S_{g}^{p}, \rho_{g}^{p} e^{i \eta_{g}^{p}}\right)$

where

$S_{g}^{p}=\left\{f \mid\right.$ if CFDP of length $\mathrm{m}$ from node $\mathrm{g}$ to node $\mathrm{f}$ exists, $\left.\vec{P}_{(g, f)}^{m}\right\}$

such that $\rho_{g}^{p}: S_{g}^{p} \rightarrow[0,1]$ given by $\rho_{g}^{p}(f)=\left\{\min \xi_{\widehat{Q}}(d, s)\right.$, $(d, s)$ is an edge of $\left.\vec{P}_{(g, f)}^{m}\right\}$ and $\eta_{g}^{p}: S_{g}^{p} \rightarrow[0,2 \pi]$ given by $\rho_{g}^{p}(f)=\left\{\min \varphi_{\widehat{Q}}(d, s),(d, s)\right.$ is an edge of $\left.\vec{P}_{(g, f)}^{m}\right\}$

Definition 25 For a node $g$ of a $\operatorname{CFG} \vec{\Omega}=(W, \widehat{\mathbb{P}}, \overrightarrow{\mathbb{Q}})$, the CF $m$-step in-neighborhood is CFS

$\aleph_{m}^{n}(g)=\left(S_{g}^{n}, \rho_{g}^{n} e^{i \eta_{g}^{n}}\right)$

where

$S_{g}^{p}=\left\{f \mid\right.$ if CFDP of length $\mathrm{m}$ from node $\mathrm{f}$ to node g exists, $\left.\vec{P}_{(f, g)}^{m}\right\}$ such that $\rho_{g}^{n}: S_{g}^{p} \rightarrow[0,1]$ given by $\rho_{g}^{n}(f)=\left\{\min \xi_{\widehat{Q}}(d, s)\right.$, $(d, s)$ is an edge of $\left.\vec{P}_{(f, g)}^{m}\right\}$ and $\eta_{g}^{n}: S_{g}^{p} \rightarrow[0,2 \pi]$ given by $\rho_{g}^{p}(f)=\left\{\min \varphi_{\widehat{\mathbb{Q}}}(d, s),(d, s)\right.$ is an edge of $\left.\vec{P}_{(f, g)}^{m}\right\}$.

Example 6 Let $\vec{\Omega}=(W, \widehat{\mathbb{P}}, \overrightarrow{\mathbb{Q}})$ be a CFDG as presented in Fig. 7, given by

$$
\widehat{\mathbb{P}}=\left\langle\frac{a}{0.8 e^{i 0.7 \pi}}, \frac{b}{1 e^{i 1.3 \pi}}, \frac{c}{0.7 e^{1.2 \pi}}, \frac{x}{0.6 e^{i 1.3 \pi}}, \frac{y}{0.5 e^{i 1.8 \pi}},\right.
$$
$\left.\frac{z}{0.9 e^{i 1.7 \pi}}\right\rangle$, 
Fig. 7 Two-step complex fuzzy competition graph of CFDG

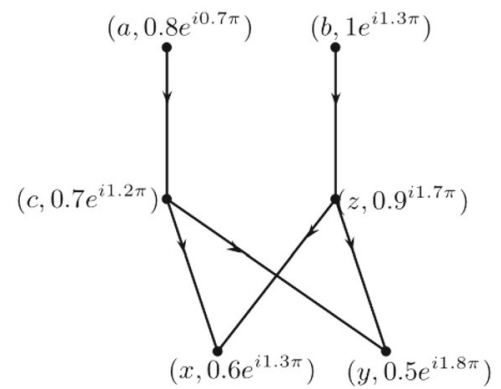

(a) CFDG $(\vec{\Omega})$

$$
\begin{aligned}
& \left(a, 0.8 \frac{\left.e^{i 0.7 \pi}\right)}{\left(0.48 e^{i 0.175 \pi}\right)}\left(b, 1 e^{i 1.3 \pi}\right)\right. \\
& \left(c, \dot{0}^{0.7 e^{i 1.2 \pi}}\right) \quad\left(z, \dot{0}^{i 1.7 \pi}\right) \\
& \left(x, \dot{0}^{i} e^{i 1.3 \pi}\right) \quad\left(y, \dot{0}^{i .5 e^{i 1.8 \pi}}\right)
\end{aligned}
$$

(b) 2-step CFCG

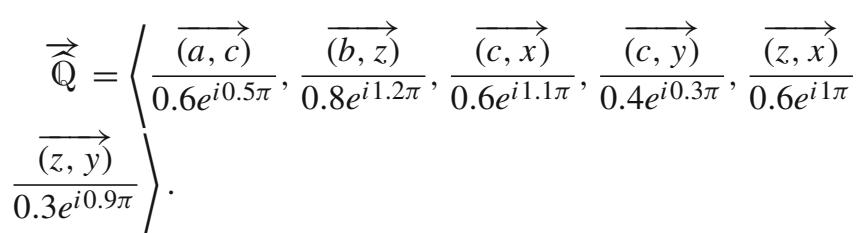

In Fig. 7a, two-step out-neighborhood of the node $a$ is the $\operatorname{CFS~}_{2}^{p}(a)=\left\{\left(x, 0.6 e^{i 0.5 \pi}\right),\left(y, 0.4 e^{i 0.3 \pi}\right)\right\}$ in $\vec{\Omega}$. Similarly, two-step in-neighborhood of the node $x$ is the CFS $\left\{\left(a, 0.6 e^{i 0.5 \pi}\right),\left(b, 0.6 e^{i 1 \pi}\right)\right\}$.

Definition 26 Let $\vec{\Omega}=(W, \widehat{\mathbb{P}}, \overrightarrow{\mathbb{Q}})$ be a CFDG. The $m$ step CFCG of $\Omega$ is symbolized by $\mathfrak{C}_{m}(\vec{\Omega})=(W, \widehat{\mathbb{P}}, \breve{\mathbb{C}})$ with same CF node set as in $\vec{\Omega}$ and possesses a CF edge joining two nodes $g$ and $f$ in $\mathfrak{C}_{m}(\vec{\Omega})$ if and only if $\aleph_{m}^{p}(g) \cap$ $\aleph_{m}^{p}(f) \neq \varnothing$ and the MV of the edge $(g, f)$ is given as

$\xi_{\breve{\mathbb{C}}}(g, f)=\left(\xi_{\widehat{\mathbb{P}}}(g) \wedge \xi_{\widehat{\mathbb{P}}}(f)\right) \times \hbar_{\xi}\left(\aleph_{m}^{p}(g) \cap \aleph_{m}^{p}(f)\right)$,

$\varphi_{\breve{\mathbb{C}}}(g, f)=2 \pi\left[\left(\frac{\varphi_{\widehat{\mathbb{P}}}(g)}{2 \pi} \wedge \frac{\varphi_{\widehat{\mathbb{P}}}(f)}{2 \pi}\right) \times \frac{\hbar_{\varphi}\left(\aleph_{m}^{p}(g) \cap \aleph_{m}^{p}(f)\right)}{2 \pi}\right]$,

for all $g, f \in W$.

Example 7 Let $\vec{\Omega}=(W, \widehat{\mathbb{P}}, \overrightarrow{\mathbb{Q}})$ be a CFDG as presented in Fig. 7a, given by

$$
\begin{aligned}
& \widehat{\mathbb{P}}=\left\langle\frac{a}{0.8 e^{i 0.7 \pi}}, \frac{b}{1 e^{i 1.3 \pi}}, \frac{c}{0.7 e^{1.2 \pi}}, \frac{x}{0.6 e^{i 1.3 \pi}}, \frac{y}{0.5 e^{i 1.8 \pi}},\right. \\
& \left.\frac{z}{0.9 e^{i 1.7 \pi}}\right\rangle, \\
& \overrightarrow{\widehat{\mathbb{Q}}}=\left\langle\frac{\overrightarrow{(a, c)}}{0.6 e^{i 0.5 \pi}}, \frac{\overrightarrow{(b, z)}}{0.8 e^{i 1.2 \pi}}, \frac{\overrightarrow{(c, x)}}{0.6 e^{i 1.1 \pi}}, \frac{\overrightarrow{(c, y)}}{0.4 e^{i 0.3 \pi}}, \frac{\overrightarrow{(z, x)}}{0.6 e^{i 1 \pi}},\right. \\
& \overrightarrow{(z, y)} \\
& \left.\overrightarrow{0.3 e^{i 0.9 \pi}}\right\rangle .
\end{aligned}
$$

The two-step out-neighborhood of the nodes are displayed in Table 9.
Table 9 Two-step out-neighborhood of the nodes

\begin{tabular}{ll}
\hline $\mathrm{g}$ & $\aleph_{2}^{p}(g)$ \\
\hline $\mathrm{a}$ & $\left\{\left(x, 0.6 e^{i 0.5 \pi}\right),\left(y, 0.4 e^{i 0.3 \pi}\right)\right\}$ \\
$\mathrm{b}$ & $\left\{\left(x, 0.6 e^{i 1.0 \pi}\right),\left(y, 0.3 e^{i 0.3 \pi}\right)\right\}$ \\
$\mathrm{c}$ & $\varnothing$ \\
$\mathrm{x}$ & $\varnothing$ \\
$\mathrm{y}$ & $\varnothing$ \\
$\mathrm{z}$ & $\varnothing$ \\
\hline
\end{tabular}

Therefore,

$\aleph_{2}^{p}(a) \cap \aleph_{2}^{p}(b)=\left\{\left(x, 0.6 e^{i 0.5 \pi}\right),\left(y, 0.3 e^{i 0.3 \pi}\right)\right\}$.

The two-step CFCG of $\vec{\Omega}$ is shown in Fig. 7b.

Now, we define the strength of CFCG below.

Definition 27 Let $\vec{\Omega}=(W, \widehat{\mathbb{P}}, \overrightarrow{\mathbb{Q}})$ be a CFDG. Let the common node of $m$-step out-neighborhoods of nodes $s_{1}, s_{2}, \cdots s_{n}$ be $x$. Also, let $\xi_{\widehat{Q}} \overrightarrow{\left(d_{1}, l_{1}\right)} e^{i \varphi_{\widehat{Q}} \overline{\left(d_{1}, l_{1}\right)}}, \xi_{\widehat{Q}} \overrightarrow{\left(d_{2}, l_{2}\right)}$ $e^{i \varphi_{\widehat{Q}}\left(\overrightarrow{\left.d_{2}, l_{2}\right)}\right.}, \cdots \xi_{\widehat{Q}} \overrightarrow{\left(d_{n}, l_{n}\right)} e^{i \varphi_{\widehat{Q}}} \overline{\left(d_{n}, l_{n}\right)}$ denote the minimum membership degrees of the edges of paths $\vec{P}_{\left(s_{1}, x\right)}^{m}, \vec{P}_{\left(s_{2}, x\right)}^{m}, \cdots$ $\vec{P}_{\left(s_{n}, x\right)}^{m}$, respectively. The $m$-step node $x \in W$ is called strong if $\xi_{\widehat{Q}} \overrightarrow{\left(d_{j}, l_{j}\right)}>\frac{1}{2}$ and $\varphi_{\widehat{Q}} \overrightarrow{\left(d_{j}, l_{j}\right)}>1 \pi$, for all $j=1,2,3, \cdots, n$.

For the prey $x$, the strength is measured by the mapping $s: W \rightarrow\{z|z \in \mathbf{C}:| z \mid \leq 1\}$ such that

$s(x)=\frac{\sum_{j=1}^{n} \xi_{\widehat{Q}} \overrightarrow{\left(d_{j}, l_{j}\right)}}{n} e^{i \frac{\sum_{j=1}^{n} \varphi_{\widehat{Q}}\left(\overrightarrow{\left.d_{j}, l_{j}\right)}\right.}{n}}$.

Example 8 Let $\vec{\Omega}=(W, \widehat{\mathbb{P}}, \overrightarrow{\mathbb{Q}})$ be a CFDG as shown in Fig. 8 , given by 


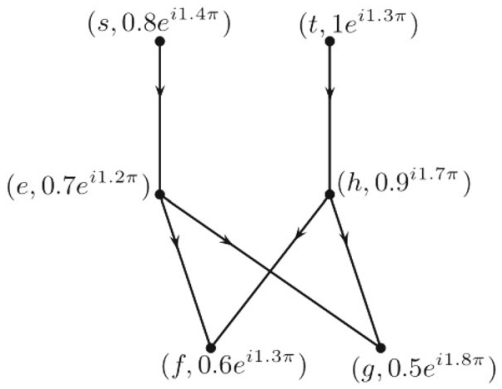

Fig. 8 Complex fuzzy digraph

$$
\begin{aligned}
& \widehat{\mathbb{P}}=\left\langle\frac{s}{0.8 e^{i 1.4 \pi}}, \frac{t}{1 e^{i 1.3 \pi}}, \frac{e}{0.7 e^{1.2 \pi}}, \frac{f}{0.6 e^{i 1.3 \pi}}, \frac{g}{0.5 e^{i 1.8 \pi}},\right. \\
& \left.\frac{h}{0.9 e^{i 1.7 \pi}}\right\rangle, \\
& \overrightarrow{\mathbb{Q}}=\left\langle\frac{\overrightarrow{(s, e)}}{0.6 e^{i 1.1 \pi}}, \frac{\overrightarrow{(t, h)}}{0.8 e^{i 1.2 \pi}}, \frac{\overrightarrow{(e, f)}}{0.6 e^{i 1.1 \pi}}, \frac{\overrightarrow{(e, g)}}{0.4 e^{i 1.2 \pi}},\right. \\
& \left.\frac{\overrightarrow{(h, f)}}{0.6 e^{i 1.1 \pi}}, \frac{\overrightarrow{(h, g)}}{0.3 e^{i 1.4 \pi}}\right\rangle .
\end{aligned}
$$

In Fig. 8, the strength of the prey $f$ is

$s(f)=\frac{0.6+0.6}{2} e^{i \frac{1.1 \pi+1.1 \pi}{2}}$.

Hence, the prey $f$ is two-step strong node as $0.6>\frac{1}{2}$ and $1.1 \pi>1 \pi$.

Theorem 6 If a node $x$ of $\vec{\Omega}$ is strong, then in strength of $x$,

$$
\begin{aligned}
& \text { 1. } \frac{\sum_{j=1}^{n} \xi_{\widehat{Q}}\left(d_{j}, l_{j}\right)}{n}>\frac{1}{2}, \\
& \text { 2. } \frac{\sum_{j=1}^{n} \varphi_{\widehat{Q}}\left(d_{j}, l_{j}\right)}{n}>1 \pi .
\end{aligned}
$$

Proof Let $\vec{\Omega}=(W, \widehat{\mathbb{P}}, \overrightarrow{\mathbb{Q}})$ be a CFDG. Let $x$ be the common node of $m$-step out-neighborhood of nodes $s_{1}, s_{2}, \cdots, s_{n}$, i.e., there exist CFDPs $\vec{P}_{\left(s_{1}, x\right)}^{m}, \vec{P}_{\left(s_{2}, x\right)}^{m}, \cdots$ $\vec{P}_{\left(s_{n}, x\right)}^{m}$, in $\vec{\Omega}$. Also, let

$$
\xi_{\widehat{Q}} \overrightarrow{\left(d_{1}, l_{1}\right)} e^{i \varphi_{\widehat{Q}} \overrightarrow{\left(d_{1}, l_{1}\right)}}, \xi_{\widehat{Q}} \overrightarrow{\left(d_{2}, l_{2}\right)} e^{i \varphi_{\widehat{Q}} \overrightarrow{\left(d_{2}, l_{2}\right)}}, \cdots \xi_{\widehat{Q}} \overrightarrow{\left(d_{j}, l_{j}\right)}
$$
$e^{i \varphi_{\widehat{Q}} \overrightarrow{\left(d_{j}, l_{j}\right)}}$ be the minimum MVs of the edges of paths $\vec{P}_{\left(a_{1}, x\right)}^{m}, \vec{P}_{\left(a_{2}, x\right)}^{m}, \cdots \vec{P}_{\left(a_{n}, x\right)}^{m}$, respectively. If $x$ is strong, each $\operatorname{arc} \overrightarrow{\left(d_{j}, l_{j}\right)}, j=1,2, \cdots, n$ is strong. So, $\xi_{\widehat{Q}} \overrightarrow{\left(d_{j}, l_{j}\right)}>$ $\frac{1}{2}$ and $\varphi_{\widehat{Q}} \overrightarrow{\left(d_{j}, l_{j}\right)}>1 \pi$. Now,

$$
s(x)=\frac{\sum_{j=1}^{n} \xi_{\widehat{Q}}\left(d_{j}, l_{j}\right)}{n} e^{i \frac{\sum_{j=1}^{n} \varphi_{\widehat{Q}}\left(d_{j}, l_{j}\right)}{n}},
$$

where

$$
\begin{aligned}
& \frac{\sum_{j=1}^{n} \xi_{\widehat{Q}}\left(d_{j}, l_{j}\right)}{n}>\frac{\frac{1}{2}+\frac{1}{2}+\cdots(n-\text { times })+\frac{1}{2}}{n}>\frac{1}{2}, \\
& \frac{\sum_{j=1}^{n} \varphi_{\widehat{Q}}\left(d_{j}, l_{j}\right)}{n}>\frac{1 \pi+1 \pi+\cdots(n-\text { times })+1 \pi}{n}>1 \pi .
\end{aligned}
$$

This completes the result.

Theorem 7 If in $\vec{\Omega}$ all the nodes are strong, then in $\mathfrak{C}_{m}(\vec{\Omega})$ all the edges are strong.

Proof Let $\vec{\Omega}=(W, \widehat{\mathbb{P}}, \overrightarrow{\mathbb{Q}})$ be a CFDG. Suppose that all the nodes of $\vec{\Omega}$ are strong. Let $\mathfrak{C}_{m}(\vec{\Omega})=(W, \widehat{\mathbb{P}}, \breve{\mathbb{C}})$ be $m$-step CFCG.

Case 1 Let $\aleph_{m}^{p}(g) \cap \aleph_{m}^{p}(f)=\varnothing$. Then there exists no edge connecting $g$ and $f$ in $\mathfrak{C}_{m}(\vec{\Omega})$. Thus, there is nothing to proof.

Case 2 Let $\aleph_{m}^{p}(g) \cap \aleph_{m}^{p}(f)$ be a non-empty set. As all the nodes are strong, then clearly $\hbar_{\xi}\left(\aleph_{m}^{p}(g) \cap \aleph_{m}^{p}(f)\right)>$ $\frac{1}{2}$ and $\hbar_{\varphi}\left(\aleph_{m}^{p}(g) \cap \aleph_{m}^{p}(f)\right)>1 \pi$ in $\vec{\Omega}$. Then the amplitude and phase term for the edge $(g, f)$ is

$$
\begin{aligned}
& \xi_{\breve{\mathbb{C}}}(g, f)=\left(\xi_{\widehat{\mathbb{P}}}(g) \wedge \xi_{\widehat{\mathbb{P}}}(f)\right) \times \hbar_{\xi}\left(\aleph_{m}^{p}(g) \cap \aleph_{m}^{p}(f)\right), \\
& \xi_{\widetilde{\mathbb{C}}}(g, f)>\left(\xi_{\widehat{\mathbb{P}}}(g) \wedge \xi_{\widehat{\mathbb{P}}}(f)\right) \times \frac{1}{2}, \\
& \varphi_{\breve{\mathbb{C}}}(g, f)=2 \pi\left[\left(\frac{\xi_{\widehat{\mathbb{P}}}(g)}{2 \pi} \wedge \frac{\xi_{\widehat{\mathbb{P}}}(f)}{2 \pi}\right) \times \frac{\hbar_{\varphi}\left(\aleph_{m}^{p}(g) \cap \aleph_{m}^{p}(f)\right)}{2 \pi}\right], \\
& \varphi_{\breve{\mathbb{C}}}(g, f)>\left[2 \pi\left(\frac{\varphi_{\widehat{\mathbb{P}}}(g)}{2 \pi} \wedge \frac{\varphi_{\widehat{\mathbb{P}}}(f)}{2 \pi}\right) \times \frac{1 \pi}{2 \pi}\right] .
\end{aligned}
$$

Then clearly $\xi_{\breve{\mathbb{C}}}(g, f)>\frac{1}{2}\left(\xi_{\widehat{\mathbb{P}}}(g) \wedge \xi_{\widehat{\mathbb{P}}}(f)\right)$ and $\varphi_{\breve{\mathbb{C}}}(g, f)>$ $\frac{1}{2}\left(2 \pi\left(\frac{\varphi_{\widehat{\mathbb{P}}}(g)}{2 \pi} \wedge \frac{\varphi_{\widehat{\mathbb{P}}}(f)}{2 \pi}\right)\right)$. Hence, the edge $(g, f)$ is strong. Since $(g, f)$ is an arbitrary edge of $\mathfrak{C}_{m}(\vec{\Omega})$, then in $\mathfrak{C}_{m}(\vec{\Omega})$ all the edges are strong.

Now, we establish a relation between $m$-step CFCG of a CFDG and CFCG of $m$-step CFDG.

Theorem 8 Let $\vec{\Omega}=(W, \widehat{\mathbb{P}}, \overrightarrow{\mathbb{Q}})$ be a CFDG. If $m>|W|$ then $\mathfrak{C}_{m}(\vec{\Omega})$ has no edges.

Proof Let $\vec{\Omega}=(W, \widehat{\mathbb{P}}, \overrightarrow{\mathbb{Q}})$ be CFDG and $\mathfrak{C}_{m}(\vec{\Omega})=$ $(W, \widehat{\mathbb{P}}, \breve{\mathbb{C}})$ be the corresponding $m$-step CFCG, where

$$
\begin{aligned}
& \xi_{\breve{\mathbb{C}}}(g, f)=\left(\xi_{\widehat{\mathbb{P}}}(g) \wedge \xi_{\widehat{\mathbb{P}}}(f)\right) \times \hbar_{\xi}\left(\aleph_{m}^{p}(g) \cap \aleph_{m}^{p}(f)\right), \\
& \varphi_{\breve{\mathbb{C}}}(g, f)=2 \pi\left[\left(\frac{\xi_{\widehat{\mathbb{P}}}}{2 \pi}(g) \wedge \frac{\xi_{\widehat{\mathbb{P}}}(f)}{2 \pi}\right) \times \frac{\hbar_{\varphi}\left(\aleph_{m}^{p}(g) \cap \aleph_{m}^{p}(f)\right)}{2 \pi}\right] .
\end{aligned}
$$


If $m>|W|$ there does not exists any CFDP of length $m$ in $\vec{\Omega}$. So, the CFS $\left(\aleph_{m}^{p}(g) \cap \aleph_{m}^{p}(f)\right)=\varnothing$, i.e., $\hbar_{\xi}\left(\aleph_{m}^{p}(g) \cap\right.$ $\left.\aleph_{m}^{p}(f)\right)=0$ and $\hbar_{\varphi}\left(\aleph_{m}^{p}(g) \cap \aleph_{m}^{p}(f)\right)=0$. Hence, there does not exist any edge in $\mathfrak{C}_{m}(\vec{\Omega})$. This proves the result.

Definition 28 Complex fuzzy $m$-step neighborhood of a node $g$ of a CFG $\Omega=(W, \widehat{\mathbb{P}}, \widehat{\mathbb{Q}})$ is the CFS

$\aleph_{m}(g)=\left(S_{g}, \rho_{g} e^{i \eta_{g}}\right)$

where

$S_{g}=\{f \mid$ if a complex fuzzy path (CFP) of length $m$

from $g$ to $f$ exists, $\left.P_{(g, f)}^{m}\right\}$

such that $\rho_{g}: S_{g} \in[0,1]$ given by $\rho_{g}(f)=\left\{\min \xi_{\widehat{\mathbb{Q}}}(a, b) \mid\right.$ $(a, b)$ is an edge of $\left.P_{(g, f)}^{m}\right\}$ and $\eta_{g}: S_{g} \in[0,2 \pi]$ given by $\eta_{g}(f)=\left\{\min \varphi_{\widehat{Q}}(a, b) \mid(a, b)\right.$ is an edge of $\left.P_{(g, f)}^{m}\right\}$.

Definition 29 Let $\vec{\Omega}=(W, \widehat{\mathbb{P}}, \overrightarrow{\mathbb{Q}})$ be CFG. The $m$-step CFNG $\vec{\Omega}$ is symbolized by $\mathfrak{N}_{m}(\Omega)=(W, \widehat{\mathbb{P}}, \breve{\mathbb{C}})$ with same CF node set as $\vec{\Omega}$ and possesses a CF edge joining two nodes $g$ and $f$ in $\mathfrak{N}_{m}(\Omega)$ if and only if CFS $\aleph_{m}(g) \cap \aleph_{m}(f) \neq \varnothing$ and the MV of the edge $(g, f)$ is calculated as

$\xi_{\breve{\mathbb{C}}}(g, f)=\left(\xi_{\widehat{\mathbb{P}}}(g) \wedge \xi_{\widehat{\mathbb{P}}}(f)\right) \times \hbar_{\xi}\left(\aleph_{m}(g) \cap \aleph_{m}(f)\right)$,

$\varphi_{\breve{\mathbb{C}}}(g, f)=2 \pi\left[\left(\frac{\varphi_{\widehat{\mathbb{P}}}(g)}{2 \pi} \wedge \frac{\varphi_{\widehat{\mathbb{P}}}(f)}{2 \pi}\right) \times \frac{\hbar_{\varphi}\left(\aleph_{m}(g) \cap \aleph_{m}(f)\right)}{2 \pi}\right]$,

for all $g, f \in W$.

Example 9 Let $\vec{\Omega}=(W, \widehat{\mathbb{P}}, \overrightarrow{\mathbb{Q}})$ be a CFG as presented in Fig. 9a, given by

$\widehat{\mathbb{P}}=\left\langle\frac{a}{0.9 e^{i 0.8 \pi}}, \frac{b}{0.8 e^{i 1.3 \pi}}, \frac{c}{0.9 e^{i 1.7 \pi}}, \frac{d}{1 e^{i 0.7 \pi}}, \frac{e}{0.6 e^{i 1.7 \pi}}\right.$, $\left.\frac{f}{0.7 e^{i 0.9 \pi}}\right\rangle$,

$\overrightarrow{\widehat{\mathbb{Q}}}=\left\langle\frac{(a, b)}{0.7 e^{i 0.7 \pi}}, \frac{(b, c)}{0.8 e^{i 1.2 \pi}}, \frac{(a, f)}{0.7 e^{i 0.7 \pi}}, \frac{(e, d)}{0.6 e^{i 0.6 \pi}}\right.$, $\left.\frac{(d, f)}{0.7 e^{i 0.6 \pi}}, \frac{(d, c)}{0.8 e^{i 0.5 \pi}}\right)$.

The CF two-step neighborhood of the nodes are displayed in Table 10.

The CFSs $\aleph(g) \cap \aleph(f)$ and $\hbar(\aleph(g) \cap \aleph(f))$ are given in Table 11.

The two-step CFNG of $\vec{\Omega}$ is shown in Fig. 9b.
Table 10 CF two-step neighborhood of the nodes

\begin{tabular}{ll}
\hline $\mathrm{g}$ & $\aleph_{2}(g)$ \\
\hline $\mathrm{a}$ & $\left\{\left(c, 0.7 e^{i 0.7 \pi}\right),\left(d, 0.7 e^{i 0.6 \pi}\right)\right\}$ \\
$\mathrm{b}$ & $\left\{\left(f, 0.7 e^{i 0.7 \pi}\right),\left(d, 0.8 e^{i 0.5 \pi}\right)\right\}$ \\
$\mathrm{c}$ & $\left\{\left(f, 0.7 e^{i 0.5 \pi}\right),\left(e, 0.6 e^{i 0.5 \pi}\right),\left(a, 0.7 e^{i 0.7 \pi}\right)\right\}$ \\
$\mathrm{d}$ & $\left\{\left(a, 0.7 e^{i 0.6 \pi}\right),\left(b, 0.8 e^{i 0.5 \pi}\right)\right\}$ \\
$\mathrm{e}$ & $\left\{\left(c, 0.6 e^{i 0.5 \pi}\right),\left(f, 0.6 e^{i 0.6 \pi}\right)\right\}$ \\
$\mathrm{f}$ & $\left\{\left(e, 0.6 e^{i 0.6 \pi}\right),\left(c, 0.7 e^{i 0.5 \pi}\right),\left(b, 0.7 e^{i 0.7 \pi}\right)\right\}$ \\
\hline
\end{tabular}

Table 11 CFSs $\aleph(g) \cap \aleph(f)$ and $\hbar(\aleph(g) \cap \aleph(f))$

\begin{tabular}{llll}
\hline $\mathrm{g}$ & $\mathrm{f}$ & $\aleph(g) \cap \aleph(f)$ & $\hbar(\aleph(g) \cap \aleph(f))$ \\
\hline $\mathrm{a}$ & $\mathrm{b}$ & $\left\{\left(d, 0.7 e^{i 0.5 \pi}\right)\right\}$ & $\left\{\left(0.7 e^{i 0.5 \pi}\right)\right\}$ \\
$\mathrm{a}$ & $\mathrm{c}$ & $\varnothing$ & $\varnothing$ \\
$\mathrm{a}$ & $\mathrm{d}$ & $\varnothing$ & $\varnothing$ \\
$\mathrm{a}$ & $\mathrm{e}$ & $\left\{\left(c, 0.6 e^{i 0.5 \pi}\right)\right\}$ & $\left\{\left(0.6 e^{i 0.5 \pi}\right)\right\}$ \\
$\mathrm{a}$ & $\mathrm{f}$ & $\left\{\left(c, 0.7 e^{i 0.5 \pi}\right)\right\}$ & $\left\{\left(0.7 e^{i 0.5 \pi}\right)\right\}$ \\
$\mathrm{b}$ & $\mathrm{c}$ & $\left\{\left(f, 0.7 e^{i 0.5 \pi}\right)\right\}$ & $\left\{\left(0.7 e^{i 0.5 \pi}\right)\right\}$ \\
$\mathrm{b}$ & $\mathrm{d}$ & $\varnothing$ & $\varnothing$ \\
$\mathrm{b}$ & $\mathrm{e}$ & $\left\{\left(f, 0.6 e^{i 0.6 \pi}\right)\right\}$ & $\left\{\left(0.6 e^{i 0.6 \pi}\right)\right\}$ \\
$\mathrm{b}$ & $\mathrm{f}$ & $\varnothing$ & $\varnothing$ \\
$\mathrm{c}$ & $\mathrm{d}$ & $\left\{\left(a, 0.7 e^{i 0.6 \pi}\right)\right\}$ & $\left\{\left(0.7 e^{i 0.6 \pi}\right)\right\}$ \\
$\mathrm{c}$ & $\mathrm{e}$ & $\left\{\left(f, 0.6 e^{i 0.5 \pi}\right)\right\}$ & $\left\{\left(0.6 e^{i 0.5 \pi}\right)\right\}$ \\
$\mathrm{c}$ & $\mathrm{f}$ & $\left\{\left(e, 0.6 e^{i 0.5 \pi}\right)\right\}$ & $\left\{\left(0.7 e^{i 0.5 \pi}\right)\right\}$ \\
$\mathrm{d}$ & $\mathrm{e}$ & $\varnothing$ & $\varnothing$ \\
$\mathrm{d}$ & $\mathrm{f}$ & $\left\{\left(b, 0.7 e^{i 0.5 \pi}\right)\right\}$ & $\left\{\left(0.7 e^{i 0.5 \pi}\right)\right\}$ \\
$\mathrm{e}$ & $\mathrm{f}$ & $\left\{\left(c, 0.6 e^{i 0.5 \pi}\right)\right\}$ & $\left\{\left(0.6 e^{i 0.5 \pi}\right)\right\}$ \\
\hline
\end{tabular}

\section{Complex fuzzy economic competition graphs}

Now a days, the transfer of money from one place to another place is very easy because of the advancement of internet. During this exchange of money, there are destinations and sources. Let us consider three projects, namely, $C_{1}, C_{2}, C_{3}$ as sources of money and let five organizations, namely $D_{1}, D_{2}, D_{3}, D_{4}, D_{5}$ in which some projects are running in (see Fig. 10). Here, the projects and organizations can be taken as vertices and interconnection between them can be illustrated by arcs. The two organizations are connected by an edge if they are under a certain project, and so on. The graph of this type is called economic competition graph. Now, we develop complex fuzzy economic competition graphs and $m$-step complex fuzzy economic competition graphs.

Definition 30 The complex fuzzy economic competition graph (CFECG) $\mathbb{E}(\vec{\Omega})$ of a CFDG $\vec{\Omega}=(W, \widehat{\mathbb{P}}, \overrightarrow{\widehat{Q}})$ is an undirected $\mathrm{CFG}$ denoted by $\Omega=(W, \widehat{\mathbb{P}}, \widehat{\mathbb{Q}})$ with same $\mathrm{CF}$ node set as in $\vec{\Omega}$ and possesses a CF edge joining two nodes 
Fig. 9 Two-step complex fuzzy neighborhood graph

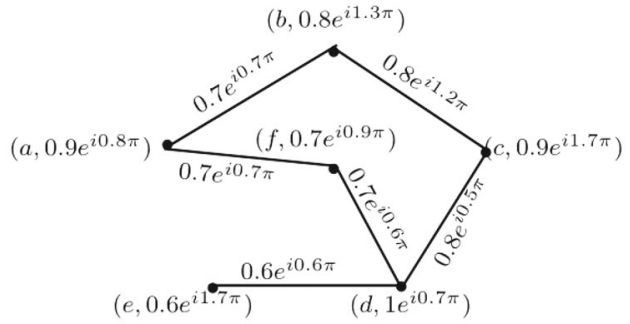

(a) A Complex fuzzy graph

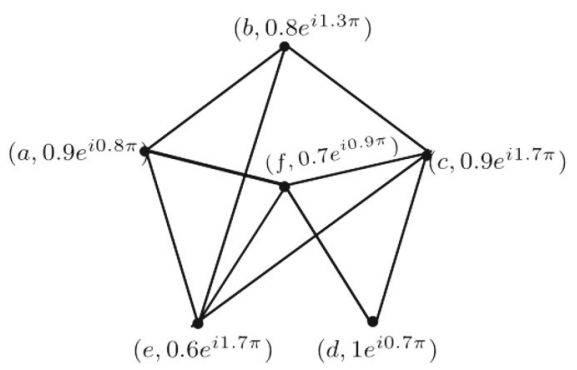

(b) $N_{2}(\Omega)$

$$
\begin{aligned}
& \xi_{\widehat{Q}}(a, b) e^{i \varphi \widehat{Q}(a, b)}=0.56 e^{i 0.2 \pi} \\
& \xi_{\widehat{Q}}(a, e) e^{i \varphi \widehat{Q}^{(a, e)}}=0.36 e^{i 0.2 \pi} \\
& \xi_{\widehat{Q}}(a, f) e^{i \varphi \widehat{Q}^{(a, f)}}=0.49 e^{i 0.2 \pi} \\
& \xi_{\widehat{Q}}(b, c) e^{i \varphi \widehat{Q}(b, c)}=0.56 e^{i 0.32 \pi} \\
& \xi_{\widehat{Q}}(b, e) e^{i \varphi \widehat{Q}^{(b, e)}}=0.36 e^{i 0.39 \pi} \\
& \xi_{\widehat{Q}}(c, d) e^{i \varphi \widehat{Q}^{(c, d)}}=0.63 e^{i 0.21 \pi} \\
& \xi_{\widehat{Q}}(c, e) e^{i \varphi \widehat{Q}^{(c, e)}}=0.36 e^{i 0.42 \pi} \\
& \xi_{\widehat{Q}}(d, f) e^{i \varphi \widehat{Q}^{(d, f)}}=0.49 e^{i 0.175 \pi} \\
& \xi_{\widehat{Q}}(e, f) e^{i \varphi \widehat{Q}^{i}(e, f)}=0.36 e^{i 0.22 \pi} \\
& \xi_{\widehat{Q}}(c, f) e^{i \varphi \widehat{Q}^{(c, f)}}=0.42 e^{i 0.22 \pi}
\end{aligned}
$$

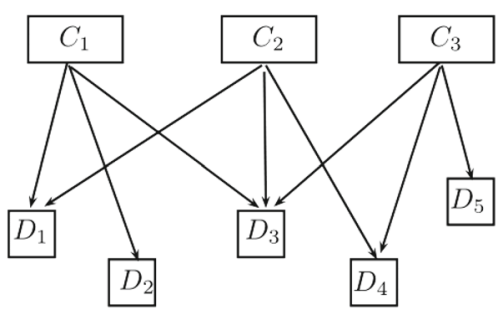

Fig. 10 Projects

$g, f \in W$ in $\mathbb{E}(\vec{\Omega})$ if and only if $\operatorname{CFS~}^{n}(g) \cap \aleph^{n}(f) \neq \varnothing$ in $\vec{\Omega}$ and the MV of the edge $(g, f)$ in $\mathbb{E}(\vec{\Omega})$ is calculated as

$$
\begin{aligned}
& \xi_{\widehat{\mathbb{Q}}}(g, f)=\left(\xi_{\widehat{\mathbb{P}}}(g) \wedge \xi_{\widehat{\mathbb{P}}}(f)\right) \times \hbar_{\xi}\left(\aleph^{n}(g) \cap \aleph^{n}(f)\right), \\
& \varphi_{\widehat{\mathbb{Q}}}(g, f)=2 \pi\left[\left(\frac{\varphi_{\widehat{\mathbb{P}}}(g)}{2 \pi} \wedge \frac{\varphi_{\widehat{\mathbb{P}}}(f)}{2 \pi}\right) \times \frac{\hbar_{\varphi}\left(\aleph^{n}(g) \cap \aleph^{n}(f)\right.}{2 \pi}\right] .
\end{aligned}
$$

Definition 31 Let $\vec{\Omega}=(W, \widehat{\mathbb{P}}, \overrightarrow{\mathbb{Q}})$ be a CFDG. The $m$ step complex fuzzy economic competition graph $(m$-step CFECG) of $\vec{\Omega}$ is denoted by $\mathbb{E}_{m}(\vec{\Omega})=(W, \widehat{\mathbb{P}}, \breve{\mathbb{C}})$ with same CF node set as in $\vec{\Omega}$ and possesses a CF edge joining two distinct nodes $g, f \in W$ in $\mathbb{E}_{m}(\vec{\Omega})$ if and only if $\aleph_{m}^{n}(g) \cap \aleph_{m}^{n}(f)$ is non-empty CFS in $\vec{\Omega}$ and the MV of the edge $(g, f)$ in $\mathbb{E}_{m}(\vec{\Omega})$ is calculated as

$$
\begin{aligned}
& \xi_{\breve{C}}(g, f)=\left(\xi_{\widehat{\mathbb{P}}}(g) \wedge \xi_{\widehat{\mathbb{P}}}(f)\right) \times, \hbar_{\xi}\left(\aleph_{m}^{n}(g) \cap \aleph_{m}^{n}(f)\right), \\
& \varphi_{\breve{\mathbb{C}}}(g, f)=2 \pi\left[\left(\frac{\varphi_{\widehat{\mathbb{P}}}(g)}{2 \pi} \wedge \frac{\varphi_{\widehat{\mathbb{P}}}(f)}{2 \pi}\right) \times \frac{\hbar_{\varphi}\left(\aleph_{m}^{n}(g) \cap \aleph_{m}^{n}(f)\right)}{2 \pi}\right],
\end{aligned}
$$

for all $g, f \in W$.

Example 10 Let $\vec{\Omega}=(W, \widehat{\mathbb{P}}, \overrightarrow{\mathbb{Q}})$ be a CFDG, where $\widehat{\mathbb{P}}=\left\langle\frac{a}{0.8 e^{i 1.7 \pi}}, \frac{b}{0.7 e^{i 1.2 \pi}}, \frac{c}{0.9 e^{i 1.1 \pi}}, \frac{d}{0.6 e^{i 0.9 \pi}}, \frac{e}{1 e^{i 0.8 \pi}}\right.$, $\left.\frac{f}{0.8 e^{i 1.3 \pi}}, \frac{g}{0.8 e^{i 1.3 \pi}}\right\rangle$

$$
\overrightarrow{\widehat{Q}}=\left\langle\frac{\overrightarrow{(a, b)}}{0.7 e^{i 1.1 \pi}}, \frac{\overrightarrow{(a, d)}}{0.6 e^{i 0.8 \pi}}, \frac{\overrightarrow{(a, f)}}{0.7 e^{i 1.2 \pi}}, \frac{\overrightarrow{(b, c)}}{0.6 e^{i 1 \pi}}, \frac{\overrightarrow{(d, c)}}{0.5 e^{i 0.8 \pi}},\right.
$$$$
\left.\frac{\overrightarrow{(d, e)}}{0.6 e^{i 0.7 \pi}}, \frac{\overrightarrow{(f, g)}}{0.6 e^{i 1.2 \pi}}, \frac{\overrightarrow{(f, e)}}{0.7 e^{i 0.6 \pi}}\right\rangle \text {. }
$$

The CFDG is presented in Fig. 11a.

The CFIN of the nodes are shown in Table 12.

The heights of CFSs $\aleph^{n}(a) \cap \aleph^{n}(b)$ and $\aleph_{2}^{n}(a) \cap \aleph_{2}^{n}(b)$ are given in Table 13.

The corresponding CFECG and two-step CFECGs are shown in Fig. $11 \mathrm{~b}$ and $\mathrm{c}$.

Theorem 9 CFECG and CFCG of any complete graphs are same. 
Fig. 11 Complex fuzzy economic competition graphs

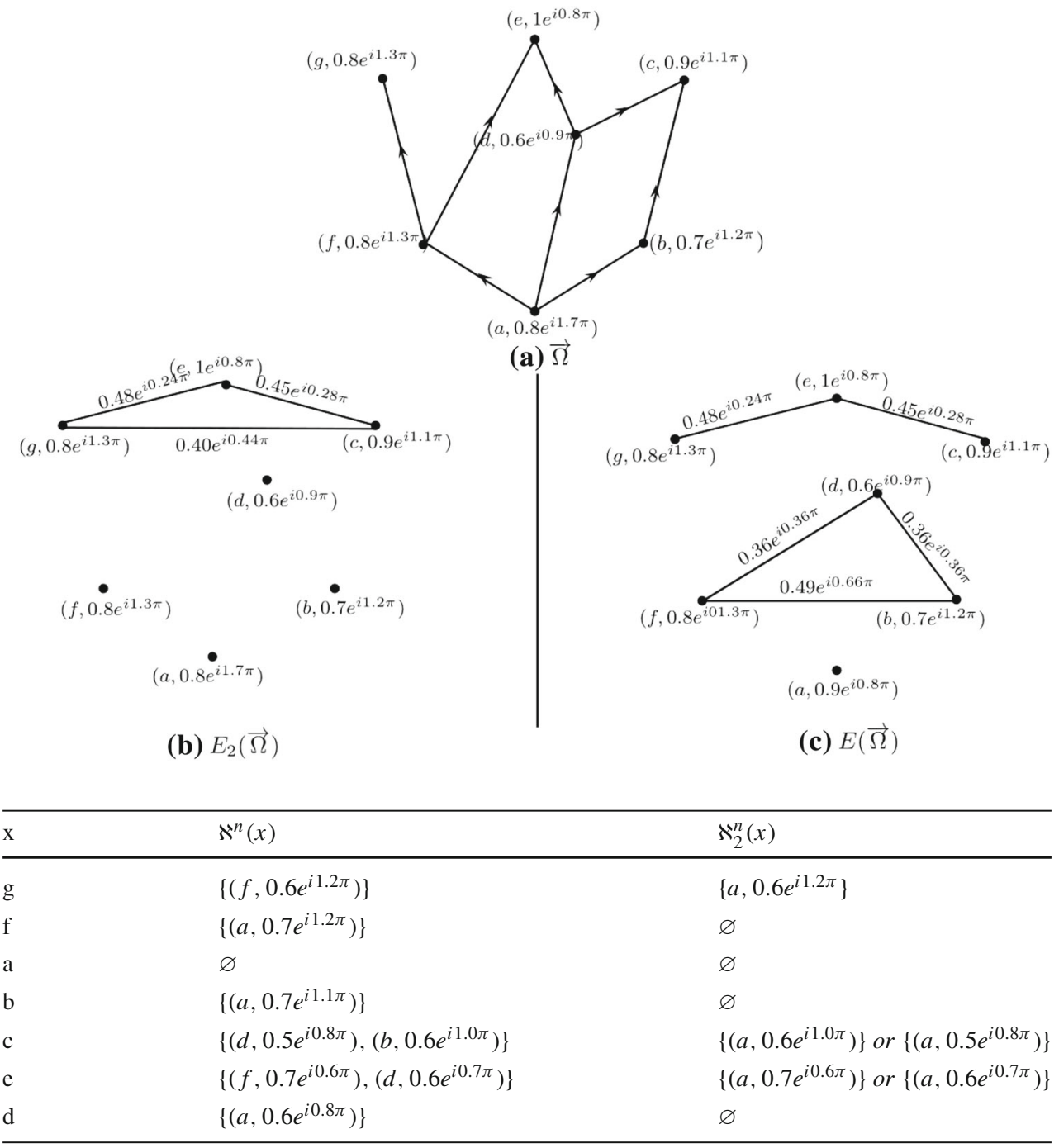

\begin{tabular}{lll}
\hline $\mathrm{x}$ & $\aleph^{n}(x)$ & $\aleph_{2}^{n}(x)$ \\
\hline $\mathrm{g}$ & $\left\{\left(f, 0.6 e^{i 1.2 \pi}\right)\right\}$ & $\left\{a, 0.6 e^{i 1.2 \pi}\right\}$ \\
$\mathrm{f}$ & $\left\{\left(a, 0.7 e^{i 1.2 \pi}\right)\right\}$ & $\varnothing$ \\
$\mathrm{a}$ & $\varnothing$ & $\varnothing$ \\
$\mathrm{b}$ & $\left\{\left(a, 0.7 e^{i 1.1 \pi}\right)\right\}$ & $\varnothing$ \\
$\mathrm{c}$ & $\left\{\left(d, 0.5 e^{i 0.8 \pi}\right),\left(b, 0.6 e^{i 1.0 \pi}\right)\right\}$ & $\left\{\left(a, 0.6 e^{i 1.0 \pi}\right)\right\}$ or $\left\{\left(a, 0.5 e^{i 0.8 \pi}\right)\right\}$ \\
$\mathrm{e}$ & $\left\{\left(f, 0.7 e^{i 0.6 \pi}\right),\left(d, 0.6 e^{i 0.7 \pi}\right)\right\}$ & $\left\{\left(a, 0.7 e^{i 0.6 \pi}\right)\right\}$ or $\left\{\left(a, 0.6 e^{i 0.7 \pi}\right)\right\}$ \\
$\mathrm{d}$ & $\left\{\left(a, 0.6 e^{i 0.8 \pi}\right)\right\}$ & $\varnothing$ \\
\hline
\end{tabular}

Table 12 CFIN of the nodes

Proof Let $\vec{\Omega}=(W, \widehat{\mathbb{P}}, \overrightarrow{\widehat{Q}})$ be a CFDG. Let $\mathfrak{C}(\vec{\Omega})=$ $(W, \widehat{\mathbb{P}}, \overrightarrow{\mathbb{Q}})$ and $\mathbb{E}(\vec{\Omega})=(W, \widehat{\mathbb{P}}, \overrightarrow{\mathbb{C}})$ are the corresponding CFCG and CFECG, respectively.

We have to show that $\mathfrak{C}(\vec{\Omega})$ and $\mathbb{E}(\vec{\Omega})$ are same. For this, we have to show that $\mathrm{CF}$ node set and CF edge set of both the graphs are same. Clearly, the CF node set of $\mathfrak{C}(\vec{\Omega})$ is same as the $\vec{\Omega}$ and also the CF node set of $\mathbb{E}(\vec{\Omega})$ is same as the $\vec{\Omega}$. Thus, the CF node set of $\mathfrak{C}(\vec{\Omega})$ is same as $\mathbb{E}(\vec{\Omega})$. Now, we need to show that $\mathrm{CF}$ edge set of $\mathfrak{C}(\vec{\Omega})$ is equal to the $\mathrm{CF}$ edge set of $\mathbb{E}(\vec{\Omega})$. The MV of the edge $(g, f)$ in $\mathfrak{C}(\vec{\Omega})$ is

$\xi_{\widehat{\mathbb{Q}}}(g, f)=\left(\xi_{\widehat{\mathbb{P}}}(g) \wedge \xi_{\widehat{\mathbb{P}}}(f)\right) \times \hbar_{\xi}\left(\aleph^{p}(g) \cap \aleph^{p}(f)\right)$,

$\varphi_{\widehat{\mathbb{Q}}}(g, f)=2 \pi\left[\left(\frac{\varphi_{\widehat{\mathbb{P}}}(g)}{2 \pi} \wedge \frac{\varphi_{\widehat{\mathbb{P}}}(f)}{2 \pi}\right) \times \frac{\hbar_{\varphi}\left(\aleph^{p}(g) \cap \aleph^{p}(f)\right)}{2 \pi}\right]$.

Also, the MV of the edge $(g, f)$ in $\mathfrak{E}(\vec{\Omega})$ is
$\xi_{\breve{\mathbb{C}}}(g, f)=\left(\xi_{\widehat{\mathbb{P}}}(g) \wedge \xi_{\widehat{\mathbb{P}}}(f)\right) \times \hbar_{\xi}\left(\aleph^{n}(g) \cap \aleph^{n}(f)\right)$,

$\varphi_{\breve{\mathbb{C}}}(g, f)=2 \pi\left[\left(\frac{\varphi_{\widehat{\mathbb{P}}}(g)}{2 \pi} \wedge \frac{\varphi_{\widehat{\mathbb{P}}}(f)}{2 \pi}\right) \times \frac{\hbar_{\varphi}\left(\aleph^{p}(g) \cap \aleph^{p}(f)\right)}{2 \pi}\right]$.

As $\vec{\Omega}$ is a complete fuzzy digraph, then clearly

$\hbar\left(\aleph^{p}(g) \cap \aleph^{p}(f)\right)=\hbar\left(\aleph^{n}(g) \cap \aleph^{n}(f)\right)$,

i.e.,

$\hbar_{\xi}\left(\aleph^{p}(g) \cap \aleph^{p}(f)\right)=\hbar_{\xi}\left(\aleph^{n}(g) \cap \aleph^{n}(f)\right)$,

$\hbar_{\varphi}\left(\aleph^{p}(g) \cap \aleph^{p}(f)\right)=\hbar_{\varphi}\left(\aleph^{n}(g) \cap \aleph^{n}(f)\right)$.

Then $\xi_{\widehat{\mathbb{Q}}}(g, f)=\xi_{\breve{\mathbb{C}}}(g, f)$ and $\varphi_{\widehat{\mathbb{Q}}}(g, f)=\varphi_{\breve{\mathbb{C}}}(g, f)$. Hence, the result is proved.

Theorem 10 If $\vec{\Omega}_{1}$ is the CF-sub-digraph of $\vec{\Omega}$. Then 
Table 13 Heights of CFSs $\aleph^{n}(a) \cap \aleph^{n}(b)$ and $\aleph_{2}^{n}(a) \cap \aleph_{2}^{n}(b)$
1. $\mathfrak{C}_{m}\left(\vec{\Omega}_{1}\right) \subset \mathfrak{C}_{m}(\vec{\Omega})$.

2. $\mathbb{E}_{m}\left(\overrightarrow{\Omega_{1}}\right) \subset \mathbb{E}_{m}(\vec{\Omega})$.

Proof Let $\vec{\Omega}=(W, \widehat{\mathbb{P}}, \overrightarrow{\mathbb{Q}})$ and $\overrightarrow{\Omega_{1}}=\left(W_{1}, \widehat{\mathbb{P}}_{1}, \widehat{\mathbb{Q}}_{1}\right)$, where $W_{1} \subset W, \xi_{\widehat{\mathbb{P}}_{1}}(g) \leq \xi_{\widehat{\mathbb{P}}}(g)$ and $\varphi_{\widehat{\mathbb{P}}_{1}}(g) \leq \varphi_{\widehat{\mathbb{P}}}(g)$ for all $g \in W_{1}$ and $\xi_{\widehat{\mathbb{Q}}_{1}} \overrightarrow{(g, f)} \leq \xi \widehat{\widehat{\mathbb{Q}}} \overrightarrow{(g, f)}$ and $\varphi_{\widehat{\mathbb{Q}}_{1}} \overrightarrow{(g, f)} \leq$ $\varphi_{\widehat{\mathbb{Q}}} \overrightarrow{(g, f)}$, for all $g, f \in W_{1}$.

1. The complex fuzzy node set of $\mathfrak{C}_{m}\left(\vec{\Omega}_{1}\right)$ is the subset of $\mathfrak{C}_{m}(\vec{\Omega})$ as $W_{1} \subset W$. Now, for any complex fuzzy edge $(g, f)$ in $\mathfrak{C}_{m}\left(\overrightarrow{\Omega_{1}}\right), \aleph_{m}^{p}(g) \cap \aleph_{m}^{p}(f)$ is the complex fuzzy subset of the same in $\mathfrak{C}_{m}(\vec{\Omega})$. So, $\xi_{\widehat{\mathbb{Q}}_{1}} \overrightarrow{(g, f)} \leq \xi_{\widehat{\mathbb{Q}}} \overrightarrow{(g, f)}$ and $\varphi_{\widehat{\mathbb{Q}}_{1}} \overrightarrow{(g, f)} \leq \varphi_{\widehat{\mathbb{Q}}} \overrightarrow{(g, f)}$ for all $g, f \in W_{1}$. Hence, this proves the result.

2. Similar to the proof 1 .

\section{Application}

Competition between objects of real world can be represented by competition graphs. However, there are some competitions of real world which cannot be represented through these competition graphs. This motivates to introduce CFCGs. In this section, we discuss an application of CFCGs to study the competition among the species of ecology.

For example, suppose a small ecosystem, sea otters like to feed on starfish and sea urchins, killer whales like to feed on sea otters, starfish want to feed on sea urchins and snails, both snails and sea urchins feed on seaweed. These six species seaweed, snail, sea urchin, starfish, sea otter and killer whale are taken as vertices. Suppose that seaweed exists $90 \%$ in the ecosystem and seaweed is $20 \%$ strong under a certain interval of time, i.e., the MV of the seaweed is $0.9 e^{i 0.4 \pi}$. Similarly, we assume the MVs of the other nodes as $0.7 e^{i 0.8 \pi}, 0.8 e^{i 1 \pi}, 0.9 e^{i 1.2 \pi}, 0.8 e^{i 1.6 \pi}, 0.9 e^{i 1.8 \pi}$, where the amplitude terms show the degree of existence of species while the phase terms show that how much the specie is strong under certain time interval. Suppose killer whale like to feed on sea otter say $60 \%$ and the prey sea otter is $70 \%$ tasty for the predator killer whale under a certain duration of time. The MV of the edge between killer whale and sea otter is $0.6 e^{i 0.7 \pi}$ and similarly the likeness and tastiness of preys for predators is shown in Table 14. The corresponding food web is shown in Fig. 12.

It is detected that if starfish are taken out from the food web then sea urchins and snails grow limitless and sea otters must be disappear. The food web can be seen by help of CFDG. 
Table 14 Likeness and tastiness of predators and preys

\begin{tabular}{llll}
\hline Name of predator & Name of prey & Like to eat $(\%)$ & Tasty to eat $(\%)$ \\
\hline Killer whale & Sea otter & 60 & 70 \\
Sea otter & Sea urchin & 60 & 45 \\
Sea otter & Starfish & 70 & 55 \\
Starfish & Sea urchin & 80 & 40 \\
Starfish & Snail & 70 & 40 \\
Snail & Seaweed & 70 & 20 \\
Sea urchin & Seaweed & 70 & 20 \\
\hline
\end{tabular}
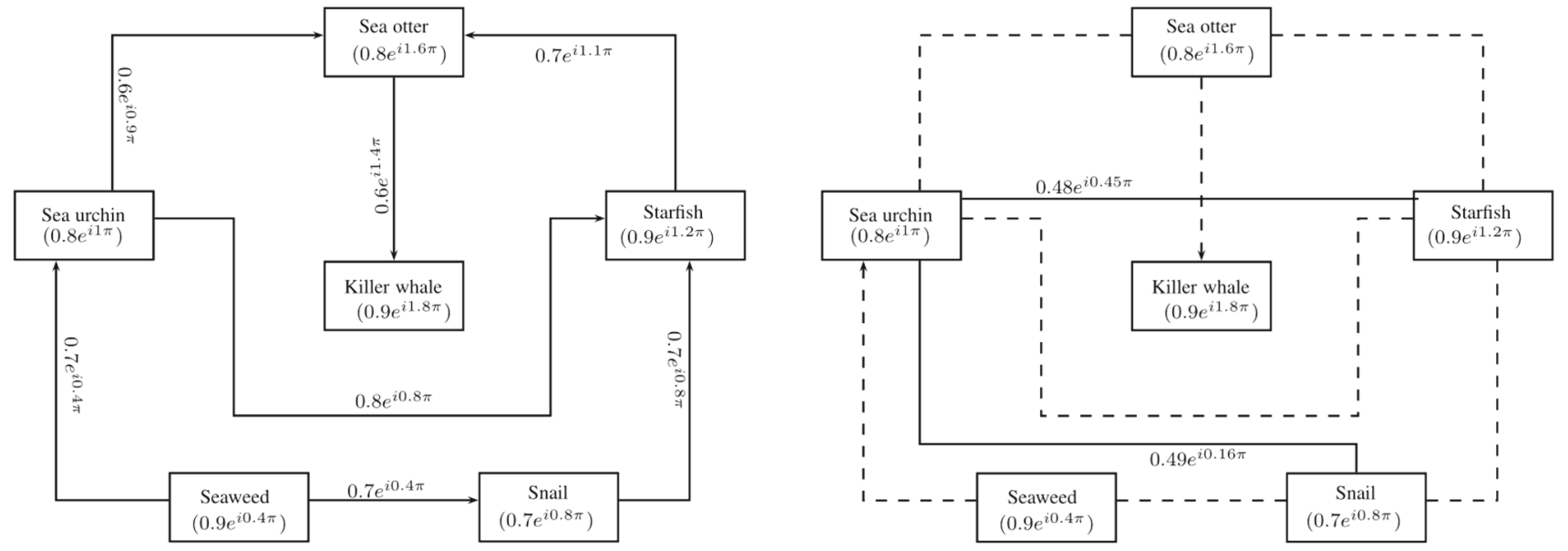

Fig. 12 Complex fuzzy digraph (food web)

Fig. 13 Complex fuzzy competition graph

Table 15 Complex fuzzy out-neighborhoods

\begin{tabular}{ll}
\hline$y \in Z$ & $\aleph^{p}(y)$ \\
\hline Killer whale & $\phi$ \\
Sea otter & $\left\{\left(\right.\right.$ killerwhale, $\left.\left.0.6 e^{i 1.4 \pi}\right)\right\}$ \\
Sea urchin & $\left\{\left(\right.\right.$ sea otter, $\left.0.6 e^{i 0.9 \pi}\right),\left(\right.$ starfish, $\left.\left.0.8 e^{i 0.8 \pi}\right)\right\}$ \\
Starfish & $\left\{\left(\right.\right.$ sea otter, $\left.\left.0.7 e^{i 1.1 \pi}\right)\right\}$ \\
Snail & $\left\{\left(\right.\right.$ starfish, $\left.\left.0.7 e^{i 0.4 \pi}\right)\right\}$ \\
Seaweed & $\left\{\left(\right.\right.$ sea urchin, $\left.0.7 e^{i 0.4 \pi}\right),\left(\right.$ snail, $\left.\left.0.7 e^{i 0.4 \pi}\right)\right\}$ \\
\hline
\end{tabular}

The CFONs of the nodes are displayed in Table 15.

There exist only two edges in CFCG as

$\aleph^{p}($ starfish $) \cap \aleph^{p}($ sea urchin $)=\left\{\left(\right.\right.$ sea otter, $\left.\left.0.6 e^{i 0.9 \pi}\right)\right\}$, $\aleph^{p}($ sea urchin $) \cap \aleph^{p}($ snail $)=\left\{\left(\right.\right.$ sea otter, $\left.\left.0.7 e^{i 0.4 \pi}\right)\right\}$.

The corresponding CFCG is shown in Fig. 13.

Now, sea urchin and starfish are connected by an edge, and similarly sea urchin and snail are connected by an edge in the CFCG. There is no edge in the CFCG for the other pair of vertices. The MVs of the edges between sea urchin and starfish is $0.48 e^{i 0.45 \pi}$, and sea urchin and snail is $0.49 e^{i 0.16 \pi}$ calculated by the definition of CFCG. Hence, sea urchin and starfish; sea urchin and snail compete for sea otter and starfish, respec- tively. Thus, there is competition between sea urchin and starfish; sea urchin and snail in the ecosystem. The method which is used in our ecosystem application is shown in Table 16 .

\section{Comparative analysis}

CGs are very useful to show the competitions among the different objects. Fuzzy competition graphs were proposed by Samanta et al. [20] to show all the competitions of the real world. Fuzzy competition graphs have larger ability to deal with vagueness and uncertainty. There are many applications of fuzzy competition graphs in various fields of life. Ecosystem is one of these applications of fuzzy competition graphs. For example, in ecology, let $\left\{\left(f_{1}, 0.9\right),\left(f_{2}, 0.8\right),\left(f_{3}, 0.9\right),\left(f_{4}, 0.8\right),\left(f_{5}, 0.7\right)\right\}$ be the set of species and let $\left\{\left(\overrightarrow{f_{2} f_{1}}, 0.7\right),\left(\overrightarrow{f_{3} f_{2}}, 0.8\right),\left(\overrightarrow{f_{5} f_{3}}, 0.7\right)\right.$, $\left.\left(\overrightarrow{f_{4} f_{3}}, 0.8\right),\left(\overrightarrow{f_{6} f_{5}}, 0.7\right)\right\}$ be the edge set, where $f_{1}, f_{2}, f_{3}, f_{4}$, $f_{5}, f_{6}$ represent the species killer whale, sea otters, urchins, starfish, snails and seaweed, respectively. The MVs of the species show the existence of these species in ecosystem and the MVs of edges show the degree of likeness of predators for the prey. These MVs of the species only show the exis- 
Table 16 Method to find competition among species

\begin{tabular}{ll}
\hline Method & Ecosystem \\
\hline Step 1 & Assign the MVs for the set of n species in the food web. $\vec{\Omega}=(\widehat{\mathbb{P}}, \overrightarrow{\mathbb{Q}})$ (say). \\
Step 2 & If for any two nodes $y_{i}$ and $y_{j}, \xi_{\widehat{\mathbb{Q}}}\left(y_{i}, y_{j}\right)>0$, then \\
& $\left(y_{j}, \xi_{\widehat{\mathbb{Q}}}\left(y_{i}, y_{j}\right) e^{i \varphi_{\widehat{Q}}\left(y_{i}, y_{j}\right)}\right) \in \aleph^{p}\left(y_{i}\right)$. \\
Step 3 & Find out the CFON $\aleph^{p}\left(y_{i}\right)$ for all vertices $y_{i}, y_{j}$. \\
Step 4 & calculate the $\aleph^{p}\left(y_{i}\right) \cap \aleph^{p}\left(y_{j}\right)$. \\
Step 5 & calculate $\hbar\left(\aleph^{p}\left(y_{i}\right) \cap \aleph^{p}\left(y_{j}\right)\right)$. \\
Step 6 & If $\aleph^{p}\left(y_{i}\right) \cap \aleph^{p}\left(y_{j}\right) \neq \varnothing$ then draw an edge $\left(y_{i}, y_{j}\right)$. \\
Step 7 & Repeat the Step 6 for all the disjoint nodes of the digraph. \\
Step 8 & Calculate the $\operatorname{MVs~using~}$ \\
& $\xi_{\widehat{Q}^{(}}\left(y_{i}, y_{j}\right)=\left(\xi_{\widehat{\mathbb{P}}}\left(y_{i}\right) \wedge \xi_{\widehat{\mathbb{Q}}} y_{j}\right) \times \hbar_{\xi}\left(\aleph^{p}\left(y_{i}\right) \cap \aleph^{p}\left(y_{j}\right)\right)$ and \\
& $\varphi_{\widehat{\mathbb{Q}}}\left(y_{i}, y_{j}\right)=2 \pi\left[\left(\frac{\varphi_{\widehat{\mathbb{P}}}\left(y_{i}\right)}{2 \pi} \wedge \frac{\varphi_{\widehat{\mathbb{P}}}\left(y_{j}\right)}{2 \pi}\right) \times \frac{\hbar_{\varphi}\left(\aleph^{p}\left(y_{i}\right) \cap \aleph^{p}\left(y_{j}\right)\right)}{2 \pi}\right]$. \\
\hline
\end{tabular}

tence of these species but it does not give the information that how much the specie is strong. Similarly, the MVs of the edges show only the likeness of preys for the predators but fails to illustrate that how much the prey is tasty to eat for the predator. This information about the preys and predators is two-dimensional which cannot be modeled by one dimensional phenomenon. This lack of knowledge motivates us to introduce the more generalized concept, CFCGs of FCGs, which have a capability to handle the two dimensional data with more perfection. The proposed work provides us a better platform to show the periodicity of the phenomenon. An application is designed in our presented work, in which the phase terms of the nodes and edges show the strength and tastiness of these species under some specific time interval. Therefore, CFCGs are more useful then the FCGs, as these graphs can handle the two-dimensional information. The aptitude of these graphs to represent the two-dimensional phenomena make it worthier to manage the ambiguous and intuitive data.

\section{Further discussion}

From the above comparison, it is clear that the proposed competition graphs are more generalized and superior the the existing competition graphs because they are efficient to deal with two-dimensional data of species in a single set. The drawbacks of the existing CGs and the main advantages of proposed CGs are given below.

1. The existing competition graphs are developed under fuzzy environment in which the uncertainties and ambiguities present in the data are handled with the help of degrees of membership that are the subset of real numbers, which may lose some useful information.

2. The proposed competition graphs are developed under $\mathrm{CF}$ environment in which the uncertainties of data are handled by complex numbers. The CFS can handle two dimensional and periodic information more precisely then the other existing models. The phase term of CFS is efficient which discriminates this set from all other existing models of the literature.

3. On removing the phase term of CFS, the CFS reduces to ordinary FS. Thus, CFS is an effective generalization of FS.

The characteristics comparison of our proposed approach with different existing approaches is given in Table 17.

\section{Conclusion}

CFS is an indispensable tool to deal with two-dimensional or periodic information due to the existence of additional term named as phase term which discriminate this set from all other existing sets of literature. In this paper, we have introduced a novel concept of CGs and economic competition graphs under $\mathrm{CF}$ environment. We have also investigated two worthwhile extensions of complex fuzzy competition graphs, named as complex fuzzy $k$-competition graphs and $p$-competition complex fuzzy graph. Moreover, we have developed neighborhood graphs and $m$-step neighborhood graphs under complex fuzzy environment with some of their remarkable results. In addition, to reveal the importance of these competition graphs in realistic situations, we have designed an application of complex fuzzy competition graphs in ecology. Finally, we have compared our proposed complex fuzzy competition graphs with existing graphs to show the superiority and authenticity of proposed graphs which leads us to the result that competition graphs are useful to represent the competition among those entities which possess the two-dimensional information. Our next interest of research is to extend our work to (1) complex fuzzy mixed graphs,

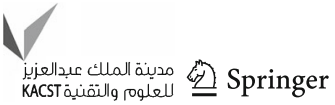


Table 17 The characteristic comparison of different graphs with existing graphs

\begin{tabular}{llll}
\hline Method & $\begin{array}{l}\text { Whether have ability } \\
\text { to handle periodic problems }\end{array}$ & $\begin{array}{l}\text { Whether have ability } \\
\text { to represent 2-D information }\end{array}$ & $\begin{array}{l}\text { Whether have the characteristics } \\
\text { of generalization }\end{array}$ \\
\hline Samanta et al. [20] & $\times$ & $\times$ & $\times$ \\
Sahoo and Pal [18] & $\times$ & $\times$ & $\times$ \\
Sarwar [21] & $\times$ & $\times$ & $\times$ \\
Habib et al. [9] & $\times$ & $\times$ & $\checkmark$ \\
The proposed CFCGs & $\checkmark$ & $\checkmark$ & $\checkmark$ \\
\hline
\end{tabular}

(2) Fermatean fuzzy competition graphs, (3) complex Fermatean fuzzy sets , (4) Fermatean fuzzy planar graphs, and (5) complex Fermatean fuzzy competition graphs.

\section{Compliance with ethical standards}

Conflict of interest The authors declare no conflict of interest.

Open Access This article is licensed under a Creative Commons Attribution 4.0 International License, which permits use, sharing, adaptation, distribution and reproduction in any medium or format, as long as you give appropriate credit to the original author(s) and the source, provide a link to the Creative Commons licence, and indicate if changes were made. The images or other third party material in this article are included in the article's Creative Commons licence, unless indicated otherwise in a credit line to the material. If material is not included in the article's Creative Commons licence and your intended use is not permitted by statutory regulation or exceeds the permitted use, you will need to obtain permission directly from the copyright holder. To view a copy of this licence, visit http://creativecomm ons.org/licenses/by/4.0/.

\section{References}

1. Akram M (2019) $m$-Polar fuzzy graphs. Stud Fuzziness Soft Comput 2019:371

2. Akram M, Luqman A (2020) Fuzzy hypergraphs and related extensions. Stud Fuzziness Soft Comput 2020:390. https://doi.org/10. 1007/978-981-15-2403-5

3. Akram M, Dudek WA, Habib A, Al-Kenani AL (2020) Imperfect competition models in economic market structure with $q$-rung picture fuzzy information. J Intell Fuzzy Syst 38(4):5107-5126

4. Brigham RC, McMorris FR, Vitray RP (1995) Tolerance competition graphs. Linear Algebra Appl 217:41-52

5. Cho HH, Kim SR, Yunsun N (2000) The $m$-step competition graph of a digraph. Discrete Appl Math 105(1-3):115-127

6. Cohen JE (1968) Interval graphs and food webs: a finding and a problem. RAND Corporation Document 17696-PR, Santa Monica, CA

7. Das SK, Panda DC, Sethi N, Gantayat SS (2011) Inductive learning of complex fuzzy relation. Int J Comput Sci Eng Inf Technol 1:2938

8. Dey A, Pal A, Long HV (2020) Fuzzy minimum spanning tree with interval type 2 fuzzy arc length: formulation and a new genetic algorithm. Soft Comput 24(6):3963-3974
9. Habib A, Akram M, Farooq A (2019) q-Rung orthopair fuzzy competition graphs with application in the soil ecosystem. Mathematics 7(1):91

10. Garg H, Rani D (2020) Generalized geometric aggregation operators based on t-norm operations for complex intuitionistic fuzzy sets and their application to decision-making. Cogn Comput 12:679-698

11. Garg H, Rani D (2020) Novel aggregation operators and ranking method for complex intuitionistic fuzzy sets and their applications to decision-making process. Artif Intell Rev 53:3595-3620

12. Isaak G, Kim SR, McKees TA, McMorris FR, Roberts FS (1992) 2-competition graphs. SIAM J Discrete Math 5(4):524-538

13. Kim SR, McKee TA, McMorris FR, Roberts FS (1995) $p$ Competition graphs. Linear Algebra Appl 217:167-178

14. Luqman A, Akram M, Al-Kenani AN, Alcantud JCR (2019) A study on hypergraph representations of complex fuzzy information. Symmetry 11(11):1381

15. Mohanta K, Dey A, Pal A, Long HV, Son LH (2020) A study of $m$-polar neutrosophic graph with applications. J Intell Fuzzy Syst 38(4):4809-4828

16. Ramot D, Milo R, Friedman M, Kandel A (2002) Complex fuzzy sets. IEEE Trans Fuzzy Syst 10(2):171-186

17. Rosenfeld A (1975) Fuzzy graphs. Fuzzy sets and their applications to cognitive and decision processes. Academic Press, Cambridge, pp 77-95

18. Sahoo S, Pal M (2016) Intuitionistic fuzzy competition graphs. J Appl Math Comput 52(1-2):37-57

19. Samanta S, Akram M, Pal M (2015) $m$-Step fuzzy competition graphs. J Appl Math Comput 47(1-2):461-472

20. Samanta S, Pal M (2013) Fuzzy $k$-competition graphs and $p$ competition fuzzy graphs. Fuzzy Inf Eng 5(2):191-204

21. Sarwar M, Akram M, Alshehri M (2018) A new method to decisionmaking with fuzzy competition hypergraphs. Symmetry 10(9):404

22. Scott DD (1987) The competition-common enemy graph of a digraph. Discrete Appl Math 17(3):269-280

23. Sonntag M, Teichert HM (2004) Competition hypergraphs. Discrete Appl Math 143(1-3):324-329

24. Thirunavukarasu P, Suresh R, Viswanathan KK (2016) Energy of a complex fuzzy graph. Int J Math Sci Eng Appl 10:243-248

25. Zadeh LA (1965) Fuzzy sets. Inf Control 8(3):338-353

26. Zhang G, Dillon TS, Cai KY, Ma J, Lu J (2009) Operation properties and d-equalities of complex fuzzy sets. Int J Approx Reason 50(8):1227-1249

27. Zuo C, Pal A, Dey A (2019) New concepts of picture fuzzy graphs with application. Mathematics 7(5):470

Publisher's Note Springer Nature remains neutral with regard to jurisdictional claims in published maps and institutional affiliations. 